\title{
Glia Maturation Factor-Gamma Regulates Autophagy and Scavenger Receptor-Mediated Phagocytosis in Murine Macrophages
}

\section{Wulin Aerbajinai}

National Institutes of Health

Jianqiong Zhu

National Institutes of Health

Kyung Chin

National Institutes of Health

Griffin Rodgers ( $\nabla$ gr5n@nih.gov )

National Institutes of Health

\section{Research Article}

Keywords: phagocytosis, critical processes, (LKB1)/AMP, AMPK, Glia maturation factor-gamma (GMFg)

Posted Date: January 20th, 2021

DOI: https://doi.org/10.21203/rs.3.rs-147914/v1

License: @ (i) This work is licensed under a Creative Commons Attribution 4.0 International License.

Read Full License 


\title{
Glia maturation factor-gamma regulates autophagy and scavenger receptor-mediated phagocytosis
} in murine macrophages

\author{
Wulin Aerbajinai ${ }^{1}$, Jianqiong Zhu ${ }^{1}$, Kyung Chin $^{1}$, and Griffin P. Rodgers ${ }^{1 *}$ \\ ${ }^{1}$ Molecular and Clinical Hematology Branch, National Heart, Lung, and Blood Institute, National \\ Institutes of Health, Bethesda, MD 20892, USA \\ *Corresponding author: Griffin P. Rodgers \\ E-mail: gr5n@nih.gov
}




\begin{abstract}
Autophagy and phagocytosis are critical processes involved in maintaining macrophage homeostasis and cellular immunity. Because dysfunction of autophagy is observed in many human pathologies, it is important to understand the regulatory mechanisms governing crosstalk between autophagy and phagocytosis. Glia maturation factor-gamma (GMF $\gamma$ ) is a novel regulator of the Arp2/3 complex, its role in modulating autophagy and phagocytosis remains unknown. Here, we show that knockdown of GMF $\gamma$ in murine macrophages inhibited autophagosome formation and compromised lysosomal function. GMF $\gamma$ knockdown suppressed phosphorylation of liver kinase B1 (LKB1)/AMP-activated protein kinase (AMPK) signaling pathway components, suggesting a role for this pathway in GMF $\gamma$ regulation of autophagy. Moreover, GMF $\gamma$-knockdown macrophages displayed increased the expression of scavenger-receptor MSR1 and CD36, which was dependent on activation of the nuclear factor erythroid 2-related factor 2 (Nrf2) transcription factor, and exhibited increased phagocytic activity. In contrast, overexpression of GMF $\gamma$ in murine macrophages increased autophagosome abundance and suppressed both scavenger-receptor expression and phagocytic activity. These findings suggest that GMF $\gamma$ regulates autophagy through AMPKregulated control of autophagosome formation, while mediating phagocytosis through modulation of scavenger-receptor abundance in macrophages, and may provide insight into therapeutic approaches to autophagy-related diseases and autophagy-regulated phagocytosis in immune and metabolic disorders.
\end{abstract}




\section{Introduction}

Autophagy and phagocytosis are evolutionarily conserved cellular processes involved in controlling key steps in innate and adaptive immunity, including inflammatory responses and bacterial infections ${ }^{1,2}$. Although autophagy and phagocytosis are commonly treated as completely separate biological phenomena, accumulated evidence has recently revealed the intrinsic connection between autophagy and phagocytosis, including newly discovered LC3-associated phagocytosis (LAP), which is a process wherein elements of autophagy conjugate LC3 to phagosomal membranes ${ }^{3}$.

Autophagy is a multistep self-digesting process mechanism that is responsible for the removal of damaged organelles, proteins malformed during their biosynthesis, and bacteria ${ }^{4,5}$. The induction of autophagy is mediated via nutrient- or energy-sensing pathways involving the inhibition of mTOR and/or the activation of AMP-activated protein kinase (AMPK) ${ }^{6}$. AMPK is ubiquitously expressed and activated in response to nutrient deprivation or other autophagy-promoting cues by multiple mechanisms, including phosphorylation by the liver kinase B1 (LKB1) in response to energy stress ${ }^{7,8}$. AMPK-mediated induction of autophagy can also bypass mTOR by directly inducing phosphorylation of ULK1 (unc-51-like kinase 1), VPS34 (vacuolar protein sorting 34), and Beclin $1^{6}$. Subsequently, ULK1 phosphorylates several downstream protein complexes, including autophagy-related (ATG) proteins and the class III PI3K (phosphoinositide 3-kinase) complex, thereby producing phosphatidylinositol 3-phosphate ${ }^{9}$. These protein complexes then lead to formation of the autophagosome, which is completed by protein conjugation and lipidation complexes that involve the conversion of the microtubule-associated protein light chain 3B-I (LC3B-I) to LC3B-II ${ }^{10}$. Finally, autophagosome fusion with a late endosome and then the lysosome produces an autolysosome, which then degrades cellular material ${ }^{11,12}$.

In contrast to autophagy, phagocytosis is a complex cellular clearance process for the capture and elimination of extracellular materials and maintenance of tissue homeostasis. Phagocytosis is commonly initiated by cell-surface receptor-mediated recognition of target particles that trigger plasma membrane and actin cytoskeleton remodeling ${ }^{13,14}$. Once the bound particle is internalized inside a phagosome vacuole, the vacuole can fuse selectively with primary lysosomes, or the vesicles coming from the endoplasmic reticulum and Golgi complex, to form a phagolysosome and subsequently degrade the internalized particle ${ }^{15}$. Phagocytic uptake of exogenous particles has features in common with the autophagic pathway, as the vacuoles used for the engulfment of extracellular materials are similar to autophagosomes, and their formation requires the core autophagy machinery. Indeed, the convergence of the autophagic and phagocytic pathways has emerged as a fundamental element in the innate immune response to invasive microbes ${ }^{16,17}$. However, the interplay of autophagy and phagocytosis is complicated by the activation of the immune response to different stimuli; for this reason, the mechanism underlying autophagy's influence on phagocytosis is still not well understood.

Glia maturation factor-gamma (GMF $\gamma$ ) is a member of actin depolymerization factor-homology domain protein family and has been shown to regulate the actin-related protein (Arp) $2 / 3$ complex and actin cytoskeleton reorganization in vivo and in vitro ${ }^{18-20}$. Arp2/3-dependent actin nucleation has been shown to be involved in the autophagy process through its participation in autophagosome formation ${ }^{21,22}$. In addition, while it has been shown that many Arp2/3-associated proteins play important roles in autophagosome biogenesis, the exact mechanism of their participation is not fully understood ${ }^{23,24}$. Because the actin cytoskeleton and its reorganization are not only necessary for proper formation of autophagosome, but also influence macrophage phagocytosis ${ }^{25,26}$, it is important to understand how manipulation of the autophagic pathway may affect phagocytic cells.

Because actin and Arp2/3 are involved in the process of autophagy, we hypothesize that the Arp2/3 regulatory protein GMF $\gamma$ might play a role in the regulation of autophagy and/or phagocytosis in macrophages. Autophagy is a tightly regulated process, and defects in autophagy have been closely associated with many human diseases, including cancer, neurodegenerative diseases, myopathy, immune 
deficiencies, and premature aging ${ }^{27}$. Furthermore, interactions between autophagy and phagocytosis may critically influence the fate of both processes.

In this study, we show that GMF $\gamma$ regulates both autophagy and phagocytosis in murine macrophages. Knockdown of GMF $\gamma$ in murine macrophages impaired autophagosome formation and increased expression of two major scavenger receptors (MSR1 and CD36), and phagocytosis, while overexpression of GMF $\gamma$ induced autophagosome formation and inhibited both scavenger-receptor expression and phagocytic activity. Knockdown of GMF $\gamma$ also promoted nuclear factor erythroid 2-related factor 2 (Nrf2) transcription factor translocation to the nucleus and upregulation cell surface expression of MSR1 and CD36 scavenger receptors on the macrophage. Our results provide new insights into the role of GMF $\gamma$ in the autophagy and phagocytosis. 


\section{Results}

Knockdown of GMF $\gamma$ in murine macrophages impairs autophagosome formation

As GMF $\gamma$ is an Arp2/3 complex regulator protein and the Arp2/3 complex has been found to regulate autophagy ${ }^{21,22}$, we were interested in examining whether GMF $\gamma$ can modulate autophagy in murine macrophages. We first examined the levels of LC3B, a classical marker of autophagosome abundance, either by immunoblotting analysis of lysates or by immunofluorescent visualization of LC3BII puncta, in GMF $\gamma$-knockdown murine macrophages. In this study, autophagy-induction conditions were produced by stimulating cells with $4 \mu \mathrm{M}$ Rapamycin for $4 \mathrm{~h}$. Knockdown of GMF $\gamma$ in RAW264.7 macrophages and bone marrow-derived macrophages (BMDMs) significantly decreased LC3BII levels under both basal and autophagy-induction conditions compared with control siRNA-transfected cells (Figures 1A-1C). Because a decrease in LC3BII levels on immunoblotting could be due to either decreased formation of autophagosomes or rapid degradation of autophagolysosomes following fusion of autophagosomes with lysosomes, we assessed the autophagic flux by treatment of GMF $\gamma$-knockdown RAW264.7 cells with the lysosomal V-ATP-ase inhibitor Bafilomycin A1 (BafA1), which blocks LC3BII/autophagosome degradation, under both basal and autophagy-induction conditions. Notably, treatment with BafA1 was able to enhance LC3BII levels in GMF $\gamma$-knockdown cells under both basal and autophagy-induction conditions, but GMF $\gamma$-knockdown cells still demonstrated lower LC3BII levels compared with control-transfected cells (Figures 1A and 1B). These data suggest that GMF $\gamma$-knockdown suppressed autophagosomal biosynthetic pathway, but did not prevent autophagic flux.

The autophagy ubiquitin-binding scaffold protein p62 (sequestosome 1/SQSTM1), which accumulates upon inhibition of autophagy ${ }^{28,29}$, was significantly increased in GMF $\gamma$-knockdown RAW264.7 macrophages and BMDMs under both basal and autophagy-induction conditions, compared with controltransfected cells (Figures 1A-1C). These results imply that the suppressed autophagy observed upon GMF $\gamma$ knockdown leads to an accumulation of p62 aggregates. Moreover, immunofluorescent analysis showed decreased punctate LC3B structures per cell in GMF $\gamma$-knockdown RAW264.7 macrophages compared with control-transfected cells under autophagy-induction conditions (Figure 1D), indicating that fewer autophagosomes were found under these conditions. Collectively, these data suggest that GMF $\gamma$ may regulate autophagy at the early stage in autophagosome formation.

Atg9A, a transmembrane protein that delivers membrane lipids to the growing autophagosome, was recently shown to be required for the actin assembly that occurs at autophagosome formation ${ }^{30,31}$. We thus investigated whether GMF $\gamma$ influences the endosomal trafficking of Atg9A during autophagosome biogenesis. Knockdown of GMFy in RAW264.7 macrophages in part decreased colocalization of Atg9A with both the early endosome marker EEA1 and the autophagosome marker LC3BII under autophagyinduction conditions (Figures $1 \mathrm{E}$ and $1 \mathrm{~F}$ ), and decreased the number of LC3BII puncta per cell, a phenomenon that correlates with reduced autophagosome biogenesis. The localization of Atg9A in GMF $\gamma-$ knockdown RAW264.7 macrophages was less scattered than what was observed in control-transfected cells under autophagy-induction conditions (Figure 1G). These data suggest that GMF $\gamma$ knockdown-suppressed autophagosome formation is associated with reduced Atg9A trafficking to early endosomes and autophagosomes.

Knockdown of GMF in murine macrophages inhibits lysosomal function

Lysosomes are the final destination of the autophagic pathway; therefore, impaired autophagosome processing can affect autophagosome-lysosome fusion or lysosome function. To assess autophagosomelysosome fusion in GMF $\gamma$-knockdown RAW264.7 macrophages, we first examined macrophage vesicles double-labeled for LC3B and Lamp1, a marker protein for late endosomal compartments and lysosomes. Knockdown of GMF $\gamma$ under basal conditions remarkably reduced the colocalization of endogenous LC3B with Lamp1, and resulted in diffuse cytoplasmic distribution of Lamp1-positive compartments, compared with control-transfected cells (Figure 2A), the latter of which is a phenomena commonly associated with 
compromised lysosomal function. We next assessed the acidity of lysosomes by fluorescence microscopy using the acidic fluorescent probe LysoTracker Red. GMF $\gamma$-knockdown cells displayed an increased LysoTracker Red staining compared with control-transfected cells under basal conditions (Figure 2B), suggesting that GMF $\gamma$-knockdown increased acidification of lysosomes correlates with the accumulated and diffuse distribution of lysosomes. Lysosomal function relies on lysosomal hydrolytic enzyme activation in an acidic environment ${ }^{32,33}$. Indeed, we observed that cathepsin D (CTSD) perinuclear staining was decreased, and CTSD was less colocalized with Lamp1-positive lysosomal compartments, under basal conditions in GMF $\gamma$-knockdown cells compared with control-transfected cells (Figure 2C). Consistent with these results, knockdown of GMF $\gamma$ diminished maturation of the lysosomal protease CTSD, resulting in increased levels of immature CTSD, compared with control-transfected cells. Similar results were observed following treatment of GMF $\gamma$-knockdown macrophages with chloroquine, which inhibits autophagosomal fusion and degradation under basal conditions (Figure 2D). These results suggest that reduced maturation of CTSD under GMF $\gamma$-knockdown conditions might derive from intracellular mistrafficking and/or impaired lysosomal function.

Knockdown of GMF $\gamma$ in murine macrophages decreases phosphorylation of LKB1/AMPK and autophagy proteins

Autophagy is tightly regulated by two well-known signaling pathways, mTOR and AMPK ${ }^{6,34}$. To determine the upstream signaling pathway involved in the suppressed autophagosome formation observed in GMF $\gamma$ knockdown macrophages, we assessed the status of the mTOR and LKB1/AMPK signaling pathways in GMF $\gamma$-knockdown RAW264.7 macrophages under basal conditions. Immunoblotting analysis showed significantly reduced phosphorylation of AMPK $\alpha$ and LKB1 (which is found upstream of AMPK) in GMF $\gamma$-knockdown macrophages compared with control-transfected cells. However, phosphorylation of the mTORC1 substrate p70-S6K in GMF $\gamma$-knockdown macrophages was similar to that observed in controltransfected cells (Figure 3A). To evaluate whether GMF $\gamma$ knockdown also affects other components of the autophagy machinery, we next analyzed the expression of known autophagy regulatory and effector proteins in GMF $\gamma$-knockdown macrophages under basal conditions. GMF $\gamma$ knockdown significantly decreased the level of several proteins, including Atg5, Atg7, Atg3, and Atg12, but did not decrease levels of Atg16L1, compared with control-transfected cells (Figure 3B). These results indicate that GMF $\gamma$ knockdown-suppressed autophagy is associated with the downregulation of the LBK1/AMPK signaling pathway and several autophagy-relevant proteins, but not activation of mTOR signaling.

Knockdown of GMF $\gamma$ in murine macrophages increases scavenger-receptor expression and phagocytosis Several recent studies have revealed that loss of the autophagy protein in murine macrophages can enhance phagocytosis by increasing expression of scavenger receptors ${ }^{16,35}$. Therefore, to determine whether GMF $\gamma$ knockdown-suppressed autophagy is involved in scavenger receptor-mediated phagocytosis in murine macrophages, we first examined the expression of two key macrophage scavenger receptors, MSR1 and CD36, in GMF $\gamma$-knockdown RAW264.7 macrophages. Using immunoblotting and flow cytometry, we found that both total cellular and cell-surface expression levels of these two scavenger receptors were increased in GMF $\gamma$-knockdown macrophages compared with control-transfected macrophages (Figures 4A-4C). Consistent with these data, mRNA levels of MSR1 and CD36 were also increased in GMF $\gamma$ knockdown cells compared with control-transfected cells (Figure 4D). These data suggested that the class A scavenger receptor MSR1 and the class B scavenger receptor CD36 increases in GMF $\gamma$-knockdown cells might enhance uptake of bacteria or modified lipoproteins. For this reason, we then investigated whether GMF $\gamma$ knockdown would cause increased uptake of unopsonized Escherichia coli or Staphylococcus aureus, as well as acetylated low-density lipoprotein (LDL), all three of which have previously been demonstrated to be phagocytosed through class A and B scavenger receptors ${ }^{36}$. Phagocytosis of $E$. coli, $S$. aureus, and acetylated LDL was measured by phagocytosis assays using fluorescently labeled bacteria or acetylated LDL followed by flow-cytometry analysis. To minimize any potential role for autophagic 
degradation in these assays, we evaluated uptake at an early timepoint (after 45 min of incubation). GMF $\gamma$ knockdown macrophages exhibited significantly increased E. coli, S. aureus, and acetylated LDL uptake compared with control-transfected cells (Figures 4E and 4F). These results indicate that GMF $\gamma$-knockdown macrophages displayed increased expression of MSR1 and CD36, which correlated with their increased phagocytic activity.

Knockdown of GMF $\gamma$ in murine macrophages promotes Nrf2 nuclear translocation and increases scavenger-receptor expression by a Nrf2-dependent mechanism

We next sought to identify the mechanism responsible for the upregulation of MSR1 and CD36 in GMF $\gamma$ knockdown macrophages. Recent studies have demonstrated that these two scavenger receptors are downstream target genes for Nrf2, a key transcriptional regulator ${ }^{16,37}$. Therefore, we examined whether Nrf2 nuclear accumulation occurred in GMF $\gamma$-knockdown RAW264.7 macrophages. GMF $\gamma$ knockdown led to increased nuclear Nrf2 protein expression and its translocation into the nucleus, when compared with control-transfected cells (Figures 5A and 5B). Because Nrf2 activity is regulated by ubiquitination ${ }^{38}$, we next investigated whether knockdown of GMF $\gamma$ could regulate Nrf2 ubiquitination. Immunoprecipitation of Nrf2 protein from transfected macrophages revealed decreased Nrf2 ubiquitination in GMF $\gamma$-knockdown macrophages compared with control-transfected cells (Figure 5C). Our data suggest that GMF $\gamma$ knockdown might promote Nrf2 translocation to the nucleus through its decreased ubiquitination.

To determine the potential role of Nrf2 in mediating the increased expression of MSR1 and CD36 scavenger receptors observed in GMF $\gamma$-knockdown macrophages (Figure 4A-D), we performed a double-knockdown of Nrf2 and GMF $\gamma$ in RAW264.7 macrophages. Nrf2 and GMF $\gamma$ knockdown efficiency were confirmed by quantitative-PCR (Q-PCR) (Figure 5D). Knockdown of Nrf2 significantly reduced the expression levels of MSR1 and CD36 mRNA, as well as its downstream target genes Gstpl and Nqol compared with controltransfected macrophages (Figure 5E). Of note, Nrf2 + GMF $\gamma$ double-knockdown studies revealed that Nrf2 knockdown significantly blocked GMF $\gamma$ knockdown-enhanced expression of MSR1 and CD36 in macrophages (Figure 5E). Moreover, the increased expression levels of Gstp1 and Nqo1 mRNA in GMF $\gamma$ knockdown cells were significantly inhibited in Nrf2 + GMF $\gamma$ double-knockdown macrophages compared with GMF $\gamma$-knockdown macrophages (Figure 5F). These results show that Nrf2 is required for GMF $\gamma$ knockdown-enhanced expression of scavenger receptors in murine macrophages. These results further support that increased Nrf2 nuclear translocation and its functional activation. Taken together, these results suggest that GMF $\gamma$-knockdown enhanced MSR1 and CD36 expression through promoting the Nrf2 nuclear translocation.

GMF $\gamma$ overexpression in murine macrophages increases autophagosome formation and decreases phagocytosis

To confirm the role of GMF $\gamma$ in autophagy and autophagy-regulated phagocytosis, we next examined the effects of GMF $\gamma$ overexpression on autophagosome abundance and phagocytosis in RAW264.7 macrophages. Immunoblotting analysis showed that GFP-tagged GMF $\gamma$ was successfully transfected into RAW264.7 macrophages (Figure 6A). GMFy overexpression was associated with increased LC3BII and decreased p62 protein levels, as well as augmented LC3BII punctate staining of autophagosomes when compared with GFP control plasmid-overexpressing cells (Figures 6A and 6B). Moreover, GMF $\gamma$ overexpression decreased total cellular protein levels of MSR1 and CD36 when compared with GFP control plasmid-transfected macrophages (Figure 6C). Flow-cytometry analysis showed significantly decreased cell-surface expression levels of MSR1 and CD36 in GMF $\gamma$-GFP-overexpressing cells compared with GFP control plasmid-transfected cells (Figures 6D and 6E). Furthermore, we found that GMF $\gamma$ overexpression significantly inhibited phagocytosis of E. coli, S. aureus, and acetylated LDL compared with GFP control plasmid-transfected cells (Figures $6 \mathrm{~F}$ and $6 \mathrm{G}$ ). Together, these results further support a role for GMF $\gamma$ in 
promoting autophagy, as well as in inhibiting phagocytosis, through downregulation of MSR1 and CD36 scavenger-receptor expression. 


\section{Discussion}

Autophagy influences macrophage functions and the innate immune response by regulating phagocytosis of pathogens, apoptotic cells, as well as maintains cell homeostasis in stressful or dangerous environments ${ }^{39}$. Although recent studies have revealed that autophagy modulates pathogen recognition and phagocytosis of invasive microbes ${ }^{3,16}$, little is known about the molecular mechanism(s) underlying actin-related protein regulation of such autophagy-associated phagocytosis. In this study, we have demonstrated the novel roles for the Arp2/3-regulator protein GMF $\gamma$ in modulating both AMPK-mediated autophagy and scavenger receptor-mediated phagocytosis. Our studies show that GMF $\gamma$-knockdown suppressed LKB1/AMPKmediated autophagosome biosynthesis associated with enhanced phagocytosis by Nrf2-mediated increased expression of scavenger receptor MSR1 and CD36, suggesting that GMF $\gamma$ may playing an important role in maintenance of the immunosurveillance function in macrophages.

The actin-regulatory proteins have been associated with different roles throughout the various steps of autophagy processes ${ }^{23,40}$. Dysregulation of actin assembly or altered actin-regulatory proteins cause impairment of autophagosomes, and the consequent reduction in autophagy results in aggregation of autophagy substrates and dysfunctional lysosomal activity. In this study, we found that the actin-regulatory protein GMF $\gamma$ is involved in autophagy in macrophages, because GMF $\gamma$-knockdown macrophages exhibited decreased autophagosome formation and increased p62 levels, while GMF $\gamma$ overexpression in macrophages was sufficient to increase autophagosome abundance and decrease p62 expression. Defects in autophagosome formation might be predicted to interfere with Atg9A endocytic trafficking ${ }^{30,41-43}$. Indeed, we observed decreased association of Atg9A with the early endosomes and autophagosomes under autophagy-induction condition in GMF $\gamma$-knockdown macrophages, suggesting the impaired sorting of Atg9A from the endoplasmic reticulum to the early endosomes and autophagosomes in these cells. In the process of autophagosome formation, LC3BII lipidation and recruitment is regulated by the upstream pathway of autophagy and various components of autophagic-machinery proteins ${ }^{10}$. We also found that expression of the autophagy-related proteins Atg5, Atg7, Atg3 and Atg12 was decreased in GMF $\gamma-$ knockdown macrophages, suggesting that GMF $\gamma$ acts at an upstream autophagy pathway. The LKB1/AMPK pathway is a key intracellular metabolic sensor that plays an important role upstream of autophagy activation ${ }^{6,34}$. Indeed, knockdown of GMF $\gamma$ exhibited reduced phosphorylation of LKB1 and AMPK $\alpha$, suggesting that GMF $\gamma$ regulates autophagy in macrophages may through the upstream LKB1/AMPK pathway. In addition to inhibition of the autophagosome formation, we observed that GMF $\gamma$ knockdown affects the lysosome functions by appeared enhancement of the Lamp1-positive compartments, increased the acidification of lysosomes and decreased the maturation of cathepsin D. It has been well established that Arp2/3 complex regulator protein and actin-nucleating factors play multiple important roles in autophagy, lysosomal fusion and vesicle trafficking as well as phagocytosis ${ }^{22,40,44,45}$. In this study, we focus to investigate the role of GMF $\gamma$ in autophagy and phagocytosis. Although our results show that GMF $\gamma$ knockdown compromised lysosome function, the detailed mechanism study of the GMF $\gamma$ in regulation of lysosome function remains to be further investigated.

Recently, a series of studies suggested that autophagosome formation and phagosome formation can be interdependent and complementary processes that play an important role in the innate immune response against invasive microbes. Indeed, several lines of evidence have revealed that inhibition or loss of autophagy enhance phagocytosis ${ }^{16,35,46,47}$, while activation of autophagy by physiological or pharmacological inducers reduced the phagocytic capacity of murine macrophages ${ }^{17,47}$. This inverse correlation of autophagy and phagocytosis is likely the result of competition for cellular resources by these two pathways. Furthermore, defective autophagy in $\operatorname{Atg}^{-/}$macrophages was found to enhance phagocytosis through increased scavenger- receptors expression, which was attributed to the accumulation of autophagy-related protein p62-induced activation of $\mathrm{Nrf2} 2^{16}$. Nrf2 interaction with autophagy has been shown to be dependent on p62 ${ }^{28}$. Indeed, increased levels of p62 or phosphorylation of p62 at Ser351 (Ser349 in humans) has been shown to enhance its binding affinity to Keap1, and competitively inhibit the 
Keap1-Nrf2 interaction, leading to Nrf2 stabilization and activation ${ }^{48}$. Further, activation of Nrf2 signaling has been shown to improve bacterial clearance by alveolar macrophages ${ }^{49}$. Consistent with these results, we have demonstrated here that GMF $\gamma$-knockdown macrophages exhibited enhanced phagocytosis which associated with increased surface expression of two key scavenger receptors (MSRI and CD36), both of which are Nrf2 target genes. Moreover, we found that knockdown of GMF $\gamma$ decreased ubiquitination of $\mathrm{Nrf} 2$ (thereby promoting its subsequent translocation into the nucleus). Furthermore, we detected the upregulation of two well-known Nrf2 target genes-Nqo1 and Gstpl-in GMF $\gamma$-knockdown macrophages. Importantly, increased in scavenger receptor abundance in GMF knockdown macrophages is required for the Nrf2. These findings, combined with previously reported results, indicate that Nrf2 plays a critical role in scavenger receptor-mediated phagocytosis in macrophage.

Scavenger receptors are a large family of immunosurveillance receptors that mediate the recognition and uptake of unopsonized pathogens, modified lipoproteins, and apoptotic host cells ${ }^{50}$. They play important roles in innate immunity as pattern-recognition receptors mediating the phagocytosis of different pathogens $^{51}$. MSR1 and CD36, two well-characterized class A and B scavenger receptors, respectively, participate in macrophage phagocytosis of Gram-negative bacteria E. coli, Gram-positive bacteria S. aureus, and modified LDL (such as acetylated LDL and oxidized LDL) ${ }^{36}$. Accordingly, we observed that knockdown of GMF $\gamma$ in macrophages increased the phagocytosis of E. coli, S. aureus, and acetylated LDL as well as the surface expression of MSR1 and CD36. Conversely, overexpression of GMF $\gamma$ reduced phagocytosis of E. coli, S. aureus, and acetylated LDL, as well as surface expression of MSR1 and CD36, in macrophages. Further, it has been shown that mice lacking MSR1 exhibited defective bacterial clearance and higher proinflammatory responses when challenged with other bacteria ${ }^{52}$. CD36-deficient mice have been found to be more susceptible to infection with $S$. aureus compared with WT mice, whereas CD36 is not a major phagocytic receptor for $E$. coli in macrophages ${ }^{53,54}$.

Collectively, our data indicate that GMF $\gamma$ knockdown of macrophages impairs the autophagosome formation pathway, but promotes the efficient phagocytic clearance of pathogens through enhanced scavenger-receptor expression, thereby contributing to macrophage function. Given the wide range of pathologies in which either heightened or inhibited autophagy has been implicated as a contributor ${ }^{27}$, uncovering potential therapeutic targets that are autophagy modulators (specifically, inhibitors) is of primary importance. Our study provides a link between cell-signaling events involving cytoskeletal regulator proteins (such as GMF $\gamma$ ) and induction of the autophagy pathway and subsequent regulation of phagocytosis. This, in turn, may provide insight into GMF $\gamma$ as a novel therapeutic target to modulate autophagy-related diseases and autophagy-regulated phagocytosis in immune and metabolic disorders. 


\section{Methods \\ Reagents}

BafA1, chloroquine and Rapamycin were obtained from Sigma-Aldrich. Alexa Fluor 488-conjugated $E$. coli (K-12 strain), pHrodo ${ }^{\mathrm{TM}}$ Green $S$. aureus Bioparticles ${ }^{\circledR}$, Alexa Fluor 488-conjugated acetylated LDL, LysoTracker Red, and Lysosensor Yellow/Blue DND-160 were purchased from Thermo Fisher Scientific. Antibodies against LC3B, p62, Atg3, Atg5, Atg7, Atg16L1, Atg12, EEA1, p-AMPK $\alpha$, AMPK $\alpha$, p-LKB1, LKB1, p-p70-S6K, and $\alpha$-tubulin (Tubulin) were obtained from Cell Signaling Technology. Antibodies against MSR1 (AF1797) and CD36 (AF2519) were purchased from R\&D Systems. Antibody against GMF (LS-C112254) was obtained from LifeSpan Biosciences. Antibodies against ubiquitin, Nrf2, Atg9A, Lamp1, and CTSD were obtained from Abcam.

\section{Mouse studies}

All the mouse experiments and protocols were approved by the National Institutes of Health Ethics Committee and the Animal Care and Use Committee of the National Heart, Lung, and Blood Institute. All the experiments were carried out in accordance with the relevant guidelines and regulations, as well as in compliance with the ARRIVE guidelines. The mice were maintained and bred in a pathogen-free facility of the National Institutes of Health (NIH; Bethesda, MD).

\section{Cell culture and treatment}

The murine macrophage RAW264.7 cell line was purchased from the American Type Culture Collection (ATCC TIB-71). Cells were cultured in Dulbecco's Modified Eagle's Medium (DMEM) containing 10\% fetal bovine serum (FBS). Bone marrow cells were isolated and differentiated to BMDMs according to the procedure described previously ${ }^{55}$. Briefly, bone-marrow cells were isolated from mouse femur and tibia specimens and incubated for 7 days in DMEM containing 10\% FBS and $50 \mathrm{ng} / \mathrm{ml}$ of murine macrophage colony-stimulating factor (R\&D Systems). BMDMs were transiently transfected at day 5 of culture.

For inhibition of late stages of autophagy, siRNA-transfected cells were treated for $4 \mathrm{~h}$ with $300 \mathrm{nM}$ of BafA1 or $10 \mu \mathrm{M}$ of chloroquine, two known autophagic inhibitors that act by blocking maturation of autophagic vacuoles and their fusion with lysosomes through distinct mechanisms. To stimulate autophagy, siRNA-transfected cells were treated for $4 \mathrm{~h}$ with $4 \mu \mathrm{M}$ Rapamycin, an mTOR inhibitor.

\section{$R N A$ interference and transfection}

RAW264.7 cells $\left(2 \times 10^{6}\right.$ cells $)$ or BMDMs $\left(1 \times 10^{6}\right.$ cells $)$ were transiently transfected for $48 \mathrm{~h}$ with an siRNA against GMF $\gamma$ (s82084) or a control negative siRNA (Ctrl siRNA \#1) (Thermo Fisher Scientific) using Nucleofector II (Amaxa Biosystems). For GMF $\gamma$ + Nrf2 double-knockdown, RAW264.7 cells $(2 \times$ $10^{6}$ cells) were transiently transfected for $24 \mathrm{~h}$ with an siRNA against Nrf2 (SR427248; ORIGENE), followed by transfection with an siRNA against GMF $\gamma$ for another $48 \mathrm{~h}$, using Nucleofector II. For GMF $\gamma$ overexpression studies, RAW264.7 cells $\left(2 \times 10^{6}\right.$ cells $)$ were transiently transfected for $24 \mathrm{~h}$ with a GMF $\gamma$ GFP (ORIGENE) or GFP control plasmid (ORIGENE) using Nucleofector II. All experiments with siRNAtransfected cells were performed 48 72 h after transfection, when GMF $\gamma$ expression levels were reduced $65 \sim 80 \%$ or Nrf2 expression level were reduced $70 \%$ in siRNA-transfected cells compared with control siRNA-transfected cells.

\section{$R T-q P C R$}

Total RNA from GMFy or control siRNA-transfected cells was isolated using the RNeasy Plus Mini kit (Qiagen) according to the manufacturer's instructions. First strand cDNA was synthesized from $1 \mu \mathrm{g}$ of total RNA using the SuperScript III First Strand Synthesis System (Thermo Fisher Scientific) following the manufacturer's instructions. Real-time qPCR was performed on the cDNA from $10 \mathrm{ng}$ of RNA on a Stratagene Mx3005 $\mathrm{P}^{\circledR}$ System (Agilent Technologies) using pre-validated TaqMan primer/probe sets (purchased as Assays-on-Demand gene expression products from Applied Biosystems; Thermo Fisher 
Scientific). RT-qPCR conditions were $10 \mathrm{~min}$ at $95^{\circ} \mathrm{C}$ and 40 cycles of $15 \mathrm{~s}$ at $95^{\circ} \mathrm{C}$, followed by $1 \mathrm{~min}$ at $60^{\circ} \mathrm{C}$. Expression data were analyzed using endogenous $18 \mathrm{~S}$ rRNA expression levels as the reference gene and were normalized using the $2^{-\Delta \mathrm{CT}}$ and $2^{-\Delta \Delta \mathrm{CT}}$ methods.

\section{Immunoblotting and immunoprecipitation analysis}

GMF $\gamma$ or control siRNA-transfected cells were lysed in RIPA buffer (Thermo Fisher Scientific) or prepared in $10 \mathrm{mM}$ Tris at $\mathrm{pH} 8.0,150 \mathrm{mM} \mathrm{NaCl}, 1 \%$ Nonidet P40, 0.1\% SDS, $10 \mathrm{mM}$ EDTA, and protease inhibitors (Sigma-Aldrich). Cellular nuclear and cytoplasmic protein fractions were isolated using an NE-PER Nuclear Cytoplasmic Extraction Reagent kit (Pierce) following the manufacturer's instructions. For each experiment, equal amounts of total protein (35 $\mu \mathrm{g}$ per lane) were separated on 4-20\% SDS-PAGE gels and transferred to polyvinylidene difluoride membranes (Millipore), blocked for 60 min with 1\% BSA, and incubated overnight with indicated primary antibodies at $4^{\circ} \mathrm{C}$. For endogenous immunoprecipitation analysis, transfected RAW264.7 macrophages were lysed in IP lysis buffer (Thermo Fisher Scientific) and incubated overnight at $4^{\circ} \mathrm{C}$ with either normal IgG (Santa Cruz Biotechnology) or Nrf2 monoclonal antibody (LifeSpan BioSciences). Dynabeads protein G (Thermo Fisher Scientific) was then added and incubated with the immunocomplexes for $3 \mathrm{~h}$ at $4^{\circ} \mathrm{C}$. The immunocomplexes-beads were washed three times in washing buffer, then the proteins eluted in $30 \mu \mathrm{l}$ SDS sample buffer and subjected to immunoblotting. For densitometric quantification analyses of blot intensities, the immunoblot bands were scanned, and quantified using the Quantity One ${ }^{\circledR}$ software (Life Science Research, Bio-Rad) and normalized to Tubulin.

\section{Flow-cytometry analysis}

For the analysis of MSR1 and CD36 cell-surface expression in GMF $\gamma$ siRNA transfected cells, cells were pretreated with a mouse Fcg RIII (CD16) antibody (R\&D Systems) to block nonspecific binding for 15 min, then incubated for 30 min at $4{ }^{\circ} \mathrm{C}$ in the dark with mouse polyclonal MSR1 antibody (R\&D Systems) or mouse polyclonal CD36 antibody (R\&D Systems) in staining buffer, followed by Alexa Fluor 488conjugated secondary antibody (Thermo Fisher Scientific). Isotypes were used as controls. For the analysis of MSR1 and CD36 cell-surface expression in GMFy-GFP overexpressed cells, cells were incubated for 30 min with mouse polyclonal MSR1 or mCD36 antibody, followed by Alexa Fluor 555- conjugated secondary antibody (Thermo Fisher Scientific). Cells were washed and subjected to flow-cytometry analysis using FACSCalibur (Becton-Dickinson), and the data analyzed using CellQuest software (BD Biosciences). Cellsurface expression of MSR1 or CD36 was quantified as the MFI of the fluorescence-positive cells detected. For evaluation of phagocytosis, phagocytosis percentage and MFI in the gated events were assessed. At least 15,000 cells were evaluated to determine surface-receptor expression or percentage of phagocytosis.

\section{Immunofluorescence microscopy and LysoTracker staining}

RAW264.7 macrophages transfected with control siRNA or GMF $\gamma$ siRNA for $48 \mathrm{~h}$ were seeded at a density of $5 \times 10^{4}$ live cells per chamber in 8-chamber MatTek live-cell dishes (Thermo Fisher Scientific). In some experiments, transfected cells were first incubated with the autophagy activator Rapamycin $(4 \mu \mathrm{M})$ for $4 \mathrm{~h}$ before being stained. Cells were fixed with $4 \%$ paraformaldehyde/PBS for $20 \mathrm{~min}$, followed by incubation in ice-cold methanol for $20 \mathrm{~min}$, then permeabilized with $0.1 \%$ Triton X-100/PBS for $8 \mathrm{~min}$. Cells were preblocked in $10 \% \mathrm{FBS} / \mathrm{PBS}$ for $1 \mathrm{~h}$, then stained with the indicated primary antibodies followed by Alexa Fluor-conjugated secondary antibodies (Thermo Fisher Scientific). Nuclear DNA was stained with 4',6diamidino-2-phenylindole (DAPI; Sigma-Aldrich) for $5 \mathrm{~min}$. Cells were examined under a Zeiss LSM880 confocal microscope equipped with 405-, 488- and 594-nm lasers and Zen 2009 imaging software, using a 63X/1.3 NA oil-immersion objective (Carl Zeiss). For LysoTracker staining, LysoTracker Red (75nM) (Thermo Fisher Scientific) was added to cells, and the cells incubated at $37^{\circ} \mathrm{C}$ for 1 hour in the dark. Cells were then visualized by confocal microscopy.

\section{Phagocytosis assay}


GMF $\gamma$ or control siRNA-transfected cells $\left(5 \times 10^{4}\right)$ were seeded in 24-well plates one day before experiments began. Cells were incubated with Alexa Fluor 488-conjugated E. coli (K-12 strain), pHrodo ${ }^{\mathrm{TM}}$ Green $S$. aureus Bioparticles ${ }^{\circledR}$, or Alexa Fluor 488 -conjugated acetylated LDL at $37^{\circ} \mathrm{C}$ for 45 min, washed, and treated with acid wash $(50 \mathrm{mM}$ glycine, $150 \mathrm{mM} \mathrm{NaCl}[\mathrm{pH} 2.5])$ to remove surface-bound particles. GFP vector or GMF $\gamma$-GFP plasmid-transfected cells were incubated with pHrodo ${ }^{\mathrm{TM}}$ Red E. coli BioParticles $^{\mathrm{TM}}$ conjugate, pHrodo $^{\mathrm{TM}}$ Red $S$. aureus Bioparticles $^{\mathrm{TM}}$, Alexa Fluor ${ }^{\mathrm{TM}}$ 594-conjugated acetylated $\mathrm{LDL}$ at $37^{\circ} \mathrm{C}$ for $45 \mathrm{~min}$, washed, treated with acid wash $(50 \mathrm{mM}$ glycine, $150 \mathrm{mM} \mathrm{NaCl}[\mathrm{pH}$ 2.5]) to remove surface-bound particles. Phagocytosis was quantified by measuring fluorescence intensity using flow cytometry as described above.

\section{Statistical analysis}

All experiments were performed in duplicate or triplicate. In the figures data are expressed as the mean \pm standard deviations of the mean (S.D.). Statistically significant differences for all experiments were assessed with the unpaired Student's $t$ test. Values of $p<0.05$ were considered statistically significant.

Data availability:

All data are contained with the article. 


\section{References}

1 Oczypok, E. A., Oury, T. D. \& Chu, C. T. It's a cell-eat-cell world: autophagy and phagocytosis. Am J Pathol 182, 612-622, doi:10.1016/j.ajpath.2012.12.017 (2013).

2 Sanjuan, M. A. \& Green, D. R. Eating for good health: linking autophagy and phagocytosis in host defense. Autophagy 4, 607-611, doi:10.4161/auto.6397 (2008).

3 Sanjuan, M. A. et al. Toll-like receptor signalling in macrophages links the autophagy pathway to phagocytosis. Nature 450, 1253-1257, doi:10.1038/nature06421 (2007).

4 Xie, Z. \& Klionsky, D. J. Autophagosome formation: core machinery and adaptations. Nat Cell Biol 9, 1102-1109, doi:10.1038/ncb1007-1102 (2007).

5 Rubinsztein, D. C. The roles of intracellular protein-degradation pathways in neurodegeneration. Nature 443, 780-786, doi:10.1038/nature05291 (2006).

6 Kim, J., Kundu, M., Viollet, B. \& Guan, K. L. AMPK and mTOR regulate autophagy through direct phosphorylation of Ulk1. Nat Cell Biol 13, 132-141, doi:10.1038/ncb2152 (2011).

7 Gurumurthy, S. et al. The Lkb1 metabolic sensor maintains haematopoietic stem cell survival. Nature 468, 659-663, doi:10.1038/nature09572 (2010).

8 Shaw, R. J. et al. The tumor suppressor LKB1 kinase directly activates AMP-activated kinase and regulates apoptosis in response to energy stress. Proc Natl Acad Sci U S A 101, 3329-3335, doi:10.1073/pnas.0308061100 (2004).

9 Papinski, D. \& Kraft, C. Regulation of Autophagy By Signaling Through the Atg1/ULK1 Complex. J Mol Biol 428, 1725-1741, doi:10.1016/j.jmb.2016.03.030 (2016).

10 Lystad, A. H. \& Simonsen, A. Mechanisms and Pathophysiological Roles of the ATG8 Conjugation Machinery. Cells 8, doi:10.3390/cells8090973 (2019).

11 Tooze, S. A., Abada, A. \& Elazar, Z. Endocytosis and autophagy: exploitation or cooperation? Cold Spring Harb Perspect Biol 6, a018358, doi:10.1101/cshperspect.a018358 (2014).

12 Simonsen, A. \& Tooze, S. A. Coordination of membrane events during autophagy by multiple class III PI3-kinase complexes. J Cell Biol 186, 773-782, doi:10.1083/jcb.200907014 (2009).

13 Levin, R., Grinstein, S. \& Canton, J. The life cycle of phagosomes: formation, maturation, and resolution. Immunol Rev 273, 156-179, doi:10.1111/imr.12439 (2016).

14 Levin, R. et al. Multiphasic dynamics of phosphatidylinositol 4-phosphate during phagocytosis. Mol Biol Cell 28, 128-140, doi:10.1091/mbc.E16-06-0451 (2017).

15 Russell, D. G. Mycobacterium tuberculosis and the intimate discourse of a chronic infection. Immunol Rev 240, 252-268, doi:10.1111/j.1600-065X.2010.00984.x (2011).

16 Bonilla, D. L. et al. Autophagy regulates phagocytosis by modulating the expression of scavenger receptors. Immunity 39, 537-547, doi:10.1016/j.immuni.2013.08.026 (2013).

17 Lima, J. G. et al. Phagocytosis is inhibited by autophagic induction in murine macrophages. Biochem Biophys Res Commun 405, 604-609, doi:10.1016/j.bbrc.2011.01.076 (2011).

18 Gandhi, M. et al. GMF is a cofilin homolog that binds Arp2/3 complex to stimulate filament debranching and inhibit actin nucleation. Curr Biol 20, 861-867, doi:10.1016/j.cub.2010.03.026 (2010).

19 Nakano, K., Kuwayama, H., Kawasaki, M., Numata, O. \& Takaine, M. GMF is an evolutionarily developed Adf/cofilin-super family protein involved in the Arp2/3 complex-mediated organization of the actin cytoskeleton. Cytoskeleton (Hoboken) 67, 373-382, doi:10.1002/cm.20451 (2010).

20 Ikeda, K. et al. Glia maturation factor-gamma is preferentially expressed in microvascular endothelial and inflammatory cells and modulates actin cytoskeleton reorganization. Circ Res 99, 424-433, doi:10.1161/01.RES.0000237662.23539.0b (2006).

21 Kast, D. J., Zajac, A. L., Holzbaur, E. L., Ostap, E. M. \& Dominguez, R. WHAMM Directs the Arp2/3 Complex to the ER for Autophagosome Biogenesis through an Actin Comet Tail Mechanism. Curr Biol 25, 1791-1797, doi:10.1016/j.cub.2015.05.042 (2015). 
22 Monastyrska, I. et al. Arp2 links autophagic machinery with the actin cytoskeleton. Mol Biol Cell 19, 1962-1975, doi:10.1091/mbc.E07-09-0892 (2008).

23 Kast, D. J. \& Dominguez, R. The Cytoskeleton-Autophagy Connection. Curr Biol 27, R318R326, doi:10.1016/j.cub.2017.02.061 (2017).

24 Alekhina, O., Burstein, E. \& Billadeau, D. D. Cellular functions of WASP family proteins at a glance. J Cell Sci 130, 2235-2241, doi:10.1242/jcs.199570 (2017).

25 Rotty, J. D. et al. Arp2/3 Complex Is Required for Macrophage Integrin Functions but Is Dispensable for FcR Phagocytosis and In Vivo Motility. Dev Cell 42, 498-513 e496, doi:10.1016/j.devcel.2017.08.003 (2017).

26 Herdoiza Padilla, E. et al. mir-124-5p Regulates Phagocytosis of Human Macrophages by Targeting the Actin Cytoskeleton via the ARP2/3 Complex. Front Immunol 10, 2210, doi:10.3389/fimmu.2019.02210 (2019).

27 Levine, B., Mizushima, N. \& Virgin, H. W. Autophagy in immunity and inflammation. Nature 469, 323-335, doi:10.1038/nature09782 (2011).

28 Katsuragi, Y., Ichimura, Y. \& Komatsu, M. p62/SQSTM1 functions as a signaling hub and an autophagy adaptor. FEBS J 282, 4672-4678, doi:10.1111/febs.13540 (2015).

29 Hou, B. et al. SQSTM1/p62 loss reverses the inhibitory effect of sunitinib on autophagy independent of AMPK signaling. Sci Rep 9, 11087, doi:10.1038/s41598-019-47597-4 (2019).

30 Yamamoto, H. et al. Atg9 vesicles are an important membrane source during early steps of autophagosome formation. $J$ Cell Biol 198, 219-233, doi:10.1083/jcb.201202061 (2012).

31 Orsi, A. et al. Dynamic and transient interactions of Atg9 with autophagosomes, but not membrane integration, are required for autophagy. Mol Biol Cell 23, 1860-1873, doi:10.1091/mbc.E11-09-0746 (2012).

32 Mindell, J. A. Lysosomal acidification mechanisms. Annu Rev Physiol 74, 69-86, doi:10.1146/annurev-physiol-012110-142317 (2012).

33 Trivedi, P. C., Bartlett, J. J. \& Pulinilkunnil, T. Lysosomal Biology and Function: Modern View of Cellular Debris Bin. Cells 9, doi:10.3390/cells9051131 (2020).

34 Egan, D. F. et al. Phosphorylation of ULK1 (hATG1) by AMP-activated protein kinase connects energy sensing to mitophagy. Science 331, 456-461, doi:10.1126/science.1196371 (2011).

35 Oh, D. S. \& Lee, H. K. Autophagy protein ATG5 regulates CD36 expression and anti-tumor MHC class II antigen presentation in dendritic cells. Autophagy 15, 2091-2106, doi:10.1080/15548627.2019.1596493 (2019).

36 Areschoug, T. \& Gordon, S. Scavenger receptors: role in innate immunity and microbial pathogenesis. Cell Microbiol 11, 1160-1169, doi:10.1111/j.1462-5822.2009.01326.x (2009).

37 Ishii, T. et al. Role of Nrf2 in the regulation of CD36 and stress protein expression in murine macrophages: activation by oxidatively modified LDL and 4-hydroxynonenal. Circ Res 94, 609616, doi:10.1161/01.RES.0000119171.44657.45 (2004).

38 Sekhar, K. R., Rachakonda, G. \& Freeman, M. L. Cysteine-based regulation of the CUL3 adaptor protein Keap1. Toxicol Appl Pharmacol 244, 21-26, doi:10.1016/j.taap.2009.06.016 (2010).

39 Germic, N., Frangez, Z., Yousefi, S. \& Simon, H. U. Regulation of the innate immune system by autophagy: monocytes, macrophages, dendritic cells and antigen presentation. Cell Death Differ 26, 715-727, doi:10.1038/s41418-019-0297-6 (2019).

40 Coutts, A. S. \& La Thangue, N. B. Regulation of actin nucleation and autophagosome formation. Cell Mol Life Sci 73, 3249-3263, doi:10.1007/s00018-016-2224-z (2016).

41 Judith, D. et al. ATG9A shapes the forming autophagosome through Arfaptin 2 and phosphatidylinositol 4-kinase IIIbeta. J Cell Biol 218, 1634-1652, doi:10.1083/jcb.201901115 (2019).

42 Moreau, K. et al. Transcriptional regulation of Annexin A2 promotes starvation-induced autophagy. Nat Commun 6, 8045, doi:10.1038/ncomms9045 (2015).

43 Mari, M. et al. An Atg9-containing compartment that functions in the early steps of autophagosome biogenesis. J Cell Biol 190, 1005-1022, doi:10.1083/jcb.200912089 (2010). 
44 Dai, A., Yu, L. \& Wang, H. W. WHAMM initiates autolysosome tubulation by promoting actin polymerization on autolysosomes. Nat Commun 10, 3699, doi:10.1038/s41467-019-11694-9 (2019).

45 King, J. S. et al. WASH is required for lysosomal recycling and efficient autophagic and phagocytic digestion. Mol Biol Cell 24, 2714-2726, doi:10.1091/mbc.E13-02-0092 (2013).

46 Gao, F. et al. Autophagy regulates testosterone synthesis by facilitating cholesterol uptake in Leydig cells. J Cell Biol 217, 2103-2119, doi:10.1083/jcb.201710078 (2018).

47 Martinet, W., Schrijvers, D. M., Timmermans, J. P., Herman, A. G. \& De Meyer, G. R. Phagocytosis of bacteria is enhanced in macrophages undergoing nutrient deprivation. FEBS J 276, 2227-2240, doi:10.1111/j.1742-4658.2009.06951.x (2009).

48 Komatsu, M. et al. The selective autophagy substrate p62 activates the stress responsive transcription factor Nrf2 through inactivation of Keap1. Nat Cell Biol 12, 213-223, doi:10.1038/ncb2021 (2010).

49 Harvey, C. J. et al. Targeting Nrf2 signaling improves bacterial clearance by alveolar macrophages in patients with COPD and in a mouse model. Sci Transl Med 3, 78ra32, doi:10.1126/scitranslmed.3002042 (2011).

50 Peiser, L. \& Gordon, S. The function of scavenger receptors expressed by macrophages and their role in the regulation of inflammation. Microbes Infect 3, 149-159, doi:10.1016/s12864579(00)01362-9 (2001).

51 Canton, J., Neculai, D. \& Grinstein, S. Scavenger receptors in homeostasis and immunity. Nat Rev Immunol 13, 621-634, doi:10.1038/nri3515 (2013).

52 Suzuki, H. et al. A role for macrophage scavenger receptors in atherosclerosis and susceptibility to infection. Nature 386, 292-296, doi:10.1038/386292a0 (1997).

53 Hoebe, K. et al. CD36 is a sensor of diacylglycerides. Nature 433, 523-527, doi:10.1038/nature03253 (2005).

54 Stuart, L. M. et al. Response to Staphylococcus aureus requires CD36-mediated phagocytosis triggered by the COOH-terminal cytoplasmic domain. J Cell Biol 170, 477-485, doi:10.1083/jcb.200501113 (2005).

55 Zhang, X., Goncalves, R. \& Mosser, D. M. The isolation and characterization of murine macrophages. Curr Protoc Immunol Chapter 14, Unit 14 11, doi:10.1002/0471142735.im1401s83 (2008). 


\section{Acknowledgments}

We thank Dr. Daniela A. Malide from the National Heart, Lung, and Blood Institute Light Microscopy Core Facility for her skillful help with confocal-microscope imaging.

\section{Author contributions}

W. A. and G.P.R. designed the study; W.A. performed most of the experiments; J. Z. contribute to the mouse experiments; J. Z. and K. C. contribute to RT-qPCR data analysis; G.P.R. funding acquisition and writing-review and editing. All authors reviewed and proofread the manuscripts.

\section{Author information}

Affiliations

Molecular and Clinical Hematology Branch, National Heart, Lung, and Blood Institute, National Institutes of Health, Bethesda, MD 20892, USA

Wulin Aerbajinai, Jianqiong Zhu, Kyung Chin, and Griffin P. Rodgers

Corresponding author

Correspondence to Griffin P. Rodgers

E-mail: gr5n@nih.gov

\section{Ethics declarations}

Competing interests

The authors declare that they have no conflicts of interest with the contents of this article. 


\section{Figure Legends}

Figure 1. GMF $\gamma$ knockdown in murine macrophages impairs autophagosome formation. (A-C) RAW264.7 macrophages or BMDMs were transfected with a control negative siRNA (Ctrl siRNA) or GMF $\gamma$ siRNA for $48 \mathrm{~h}$. To induce autophagy, cells were stimulated with $4 \mu \mathrm{M}$ Rapamycin for $4 \mathrm{~h}$. Representative immunoblotting analysis of LC3B and p62 protein expression in lysates of RAW264.7 macropha treated with or without $300 \mathrm{nM}$ BafA1 for $4 \mathrm{~h}$ under basal (A) or autophagy-induction conditions (B) or BMDMs under basal and autophagy-induction conditions (C). Tubulin was used as a loading control. The graphs (lower) show relative quantification of LC3BII and p62 intensity from densitometric scans after normalizing to the control Tubulin in immunoblot (upper). Data represent the mean \pm S.D. of at least three independent experiments. Values for Ctrl siRNA-transfected cells were set to $1 .{ }^{*}, p<0.05$. (D) Representative immunofluorescence images of LC3B staining in Ctrl siRNA- or GMF $\gamma$ siRNA-transfected RAW264.7 macrophages under autophagy-induction conditions. Nuclear DNA was labeled with DAPI (blue). Images are representative of three independent experiments. Scale bars, $10 \mu \mathrm{m}$. (E and F) Representative immunofluorescence images of colocalization of Atg9A (anti-mouse Alexa Fluor 488) with EEA1 (anti-rabbit Alexa Fluor 568) under autophagy-induction conditions (E) or Atg9A (anti-mouse Alexa Fluor 488) with LC3B (anti-rabbit Alexa Fluor 568) under autophagy-induction conditions (F) in Ctrl siRNA- or GMF $\gamma$ siRNA-transfected RAW264.7 macrophages. Nuclear DNA was labeled with DAPI (blue). Images are representative of three independent experiments. Boxed areas in middle column of images in $(\mathbf{E})$ are presented as enlarged images $(2.5$-fold) in the right-hand column. Scale bars, $10 \mu \mathrm{m}$. (G) Representative immunofluorescence images of Atg9A localization in Ctrl siRNA- or GMFy siRNAtransfected RAW264.7 macrophages under autophagy-induction conditions. Autophagy-induction conditions were produced by stimulating cells with $4 \mu \mathrm{M}$ Rapamycin for $4 \mathrm{~h}$. Nuclear DNA was labeled with DAPI (blue). Images are representative of three independent experiments. Scale bars, $10 \mu \mathrm{m}$.

Figure 2. GMF $\gamma$ knockdown in murine macrophages inhibits lysosomal function. RAW264.7 macrophages were transfected with a control negative siRNA (Ctrl siRNA) or GMF $\gamma$ siRNA for 48 h. (A) Representative immunofluorescence images of colocalization of LC3B (anti-mouse Alexa Fluor 488) with Lamp 1 (anti-rabbit Alexa Fluor 568) in Ctrl siRNA- or GMF $\gamma$ siRNA-transfected macrophages under basal conditions. Nuclear DNA was labeled with DAPI (blue). Images are representative of three independent experiments. Scale bars, $10 \mu \mathrm{m}$. (B) Representative fluorescence images of Ctrl siRNA- or GMF $\gamma$ siRNAtransfected cells treated with $75 \mathrm{nM}$ LysoTracker Red for $1 \mathrm{~h}$ under basal conditions. Images are representative of three independent experiments. Scale bars, $10 \mu \mathrm{m}$. (C) Representative immunofluorescence images of colocalization of CTSD (anti-mouse Alexa Fluor 488) with Lamp1 (antirabbit Alexa Fluor 568) in Ctrl siRNA- or GMF $\gamma$ siRNA-transfected macrophages under basal conditions. Nuclear DNA was labeled with DAPI (blue). Images are representative of three independent experiments. Scale bars, $10 \mu \mathrm{m}$. (D) Representative immunoblotting analysis of CTSD maturation in lysates of Ctrl siRNA- or GMF $\gamma$ siRNA-transfected macrophages treated with or without $10 \mu \mathrm{M}$ chloroquine for $4 \mathrm{~h}$ under basal conditions. Left lane, molecular weight markers. Representative image from three independent experiments. Tubulin was used as a loading control.

Figure 3. GMF $\gamma$ knockdown in murine macrophages decreases phosphorylation of LKB1/AMPK and autophagy proteins. RAW264.7 macrophages were transfected with a control negative siRNA (Ctrl siRNA) or GMF $\gamma$ siRNA for $48 \mathrm{~h}$. Representative immunoblotting analysis of LKB1/AMPK signaling pathway (A) and autophagy-related (B) protein expression in lysates of Ctrl siRNA- or GMF $\gamma$ siRNAtransfected macrophages under basal conditions. Tubulin was used as a loading control. Representative image from three independent experiments. Lower panels, histogram showing densitometry of the indicated protein levels relative to Tubulin (control) in Ctrl siRNA- or GMF $\gamma$ siRNA-transfected cells. Data represent the mean \pm S.D. of three independent experiments. Values for Ctrl siRNA-transfected cells were set to 1 . $*, p<0.05$ compared with control cells. 
Figure 4. GMFy knockdown in murine macrophages increases scavenger-receptor expression and phagocytosis. RAW264.7 macrophages were transfected with a control negative siRNA (Ctrl siRNA) or GMF $\gamma$ siRNA for $48 \mathrm{~h}$. (A and B) Flow-cytometry analysis of cell-surface protein expression of MSR1 and CD36 in Ctrl siRNA- or GMF $\gamma$ siRNA-transfected macrophages. Representative histogram (A) and quantitative data (B) are shown. Data represent the mean \pm S.D. of three independent experiments. ${ }^{* *}, p<$ 0.01 compared with control cells. (C) Representative immunoblotting analysis of total cell protein expression of MSR1 and CD36 in lysates of Ctrl siRNA- or GMF $\gamma$ siRNA-transfected macrophages. Tubulin was used as a loading control. Representative image from three independent experiments. (D) QPCR analysis of MSR1 and CD36 mRNA expression in Ctrl siRNA- or GMF $\gamma$ siRNA-transfected macrophages. Data are expressed as fold increase, normalized to $18 \mathrm{~S}$ mRNA, and represent the mean \pm S.D. of three independent experiments. Values for Ctrl siRNA-transfected cells were set to $1 .{ }^{* *}, p<0.01$ compared with control cells. (E and F) Phagocytosis of E. coli, S. aureus, and acetylated LDL (acLDL) in Ctrl siRNA- or GMF $\gamma$ siRNA-transfected macrophages. Flow cytometry analyzes phagocytic uptake of Alexa Fluor 488-conjugated E. coli (K-12 strain), pHrodo ${ }^{\text {TM }}$ Green $S$. aureus Bioparticles ${ }^{\circledR}$, or Alexa Fluor 488-conjugated acetylated LDL after $45 \mathrm{~min}$. Representative histogram (E) and quantitative data (F) are shown. Data represent the mean phagocytosis percentage \pm S.D. of three independent experiments. ${ }^{* *}, p<$ 0.01 compared with control cells.

Figure 5. GMF $\gamma$ knockdown in murine macrophages promotes Nrf2 nuclear translocation and increases scavenger-receptor expression by a Nrf2-dependent mechanism. (A-C) RAW264.7 macrophages were transfected with a control negative siRNA (Ctrl siRNA) or GMF $\gamma$ siRNA for 48 h. (A) Representative immunoblotting analysis of $\mathrm{Nrf} 2$ protein expression in cytosolic and nuclear extracts of Ctrl siRNA- or GMF $\gamma$ siRNA-transfected macrophages. Tubulin were used as a loading control. Representative image from three independent experiments. (B) Representative immunofluorescence images of Nrf2 nuclear translocation in Ctrl siRNA- or GMF $\gamma$ siRNA-transfected macrophages. Images are representative of three independent experiments. Scale bar, $10 \mu \mathrm{m}$. (C) Representative immunoprecipitation analysis of Nrf2 ubiquitination in Ctrl siRNA- or GMF $\gamma$ siRNA-transfected macrophages. Endogenous Nrf2 was immunoprecipitated (IP) from transfected cell lysates with anti-Nrf2 (or nonspecific IgG, as a negative control) and examined for ubiquitination by immunoblotting (IB) with anti-ubiquitin or anti-Nrf2 antibodies. Representative image from two independent experiments. (D-F) RAW264.7 macrophages were transfected with a Ctrl siRNA, GMF $\gamma$ siRNA, or Nrf2 siRNA for $48 \mathrm{~h}$. or, for GMF $\gamma+\mathrm{Nrf2}$ double-knockdown, with Nrf2 siRNA for $24 \mathrm{~h}$ followed by transfection with GMF $\gamma$ siRNA for another $48 \mathrm{~h}$. Q-PCR analysis of GMF $\gamma$ and Nrf2 (D), MSR1 and CD36 (E), or Gstp1 and Nqo1 (F) mRNA expression in Ctrl siRNA-, GMF $\gamma$ siRNA-, Nrf2 siRNA-, or Nrf2 siRNA + GMF $\gamma$ siRNA-transfected macrophages. Data are expressed as fold increase, normalized to $18 \mathrm{~S}$ mRNA, and represent the mean \pm S.D. of three independent experiments. Values for Ctrl siRNA-transfected cells were set to $1 . * * p<0.01$.

Figure 6. GMF $\gamma$ overexpression in murine macrophages increases autophagosome formation and decreases phagocytosis. RAW264.7 macrophages were transfected with GMF $\gamma$-GFP or GFP control plasmid for $24 \mathrm{~h}$. (A) Representative immunoblotting analysis of GMF $\gamma$-GFP, GMF $\gamma$, LC3B, and p62 protein expression in lysates of GFP control plasmid- or GMFy-overexpressing macrophages. Representative image from three independent experiments. Tubulin was used as a loading control. (B) Representative immunofluorescence images of LC3B staining in GFP control plasmid- or GMF $\gamma$ overexpressing macrophages. Nuclear DNA was labeled with DAPI (blue). Images are representative of three independent experiments. Scale bar, $10 \mu \mathrm{m}$. (C) Representative immunoblotting analysis of MSR1 and CD36 protein expression in lysates of GFP control plasmid- or GMFy-overexpressing macrophages. Representative image from three independent experiments. Tubulin was used as a loading control. (D and E) Flow-cytometry analysis of cell-surface expression levels of MSR1 and CD36 in GFP control plasmidor GMF $\gamma$-overexpressing macrophages. Representative histogram (D) and quantitative data (E) are shown. 
Data represent the mean \pm S.D. of three independent experiments. ${ }^{* *}, p<0.01$ compared with GFP control plasmid cells. (F and $\mathbf{G}$ ) Phagocytosis of E. coli, S. aureus, and acetylated LDL (acLDL) in GFP control plasmid- or GMF $\gamma$-GFP-overexpressing macrophages. Flow cytometry analyzes phagocytic uptake of pHrodo $^{\mathrm{TM}}$ Red E. coli Bioparticles ${ }^{\mathrm{TM}}$, pHrodo $^{\mathrm{TM}}$ Red S. aureus Bioparticles ${ }^{\mathrm{TM}}$, or Alexa Fluor 594conjugated acetylated LDL after $45 \mathrm{~min}$. Representative histogram $(\mathbf{F})$ and quantitative data $(\mathbf{G})$ are shown. Data represent the mean phagocytosis percentage \pm S.D. of three independent experiments. ${ }^{* *}, p<0.01$ compared with GFP control plasmid cells. 
A. Basal conditions B.

SiRNA: Ctrl GMFy Ctrl GMFy kDa

LC3B II $20=\frac{-19}{-14}$
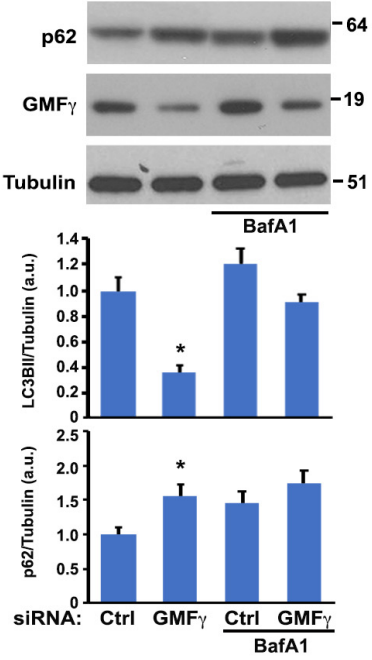

B.
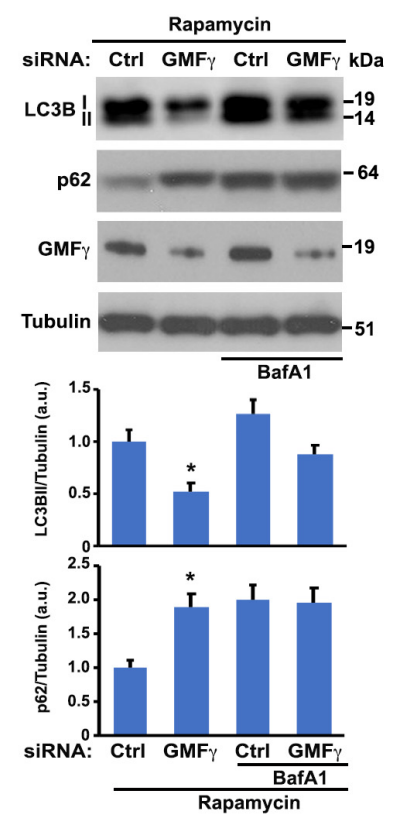

F. c.
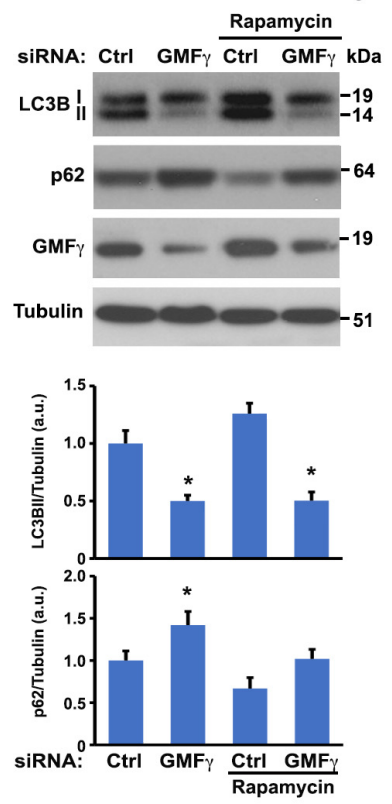

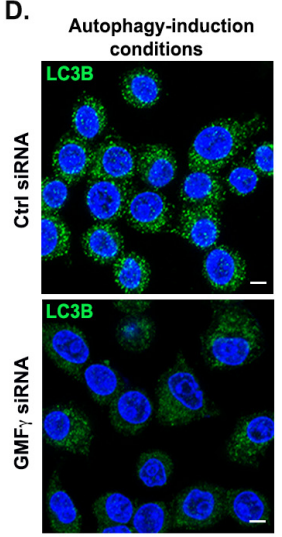

E. Autophagy-induction conditions

Autophagy-induction
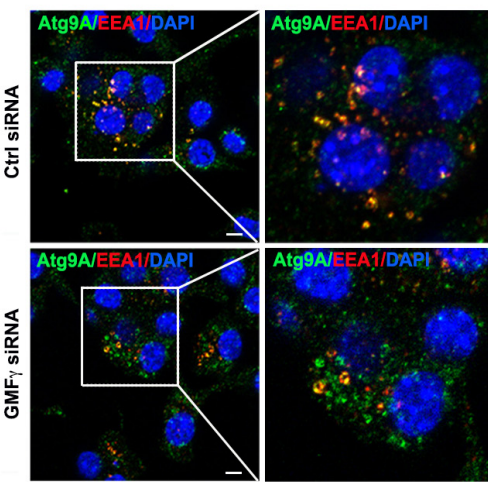

G.

Autophagy-induction conditions

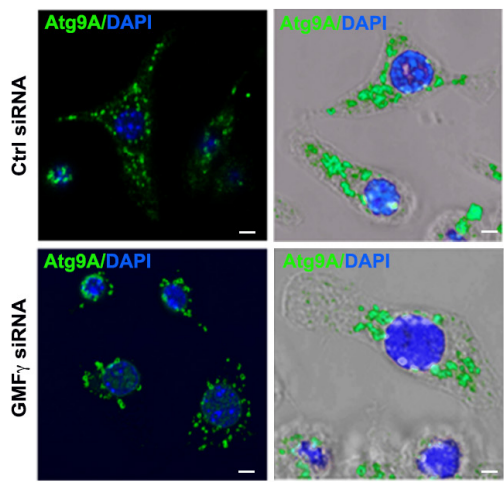


A.
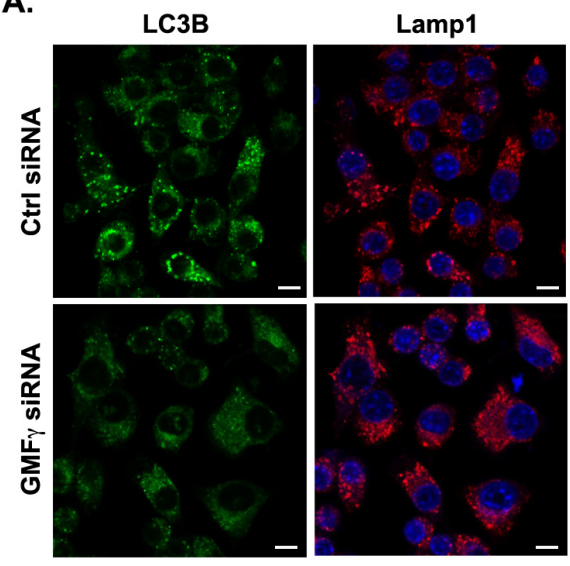

c.

C.

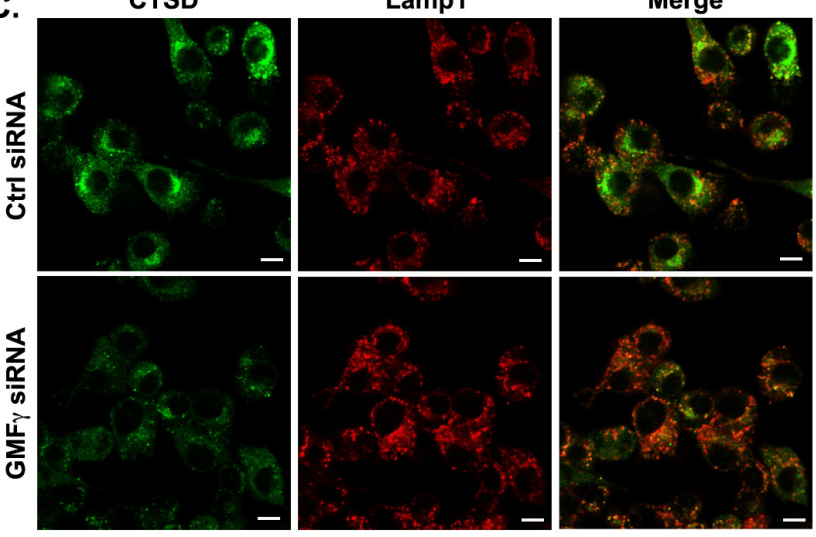

B.

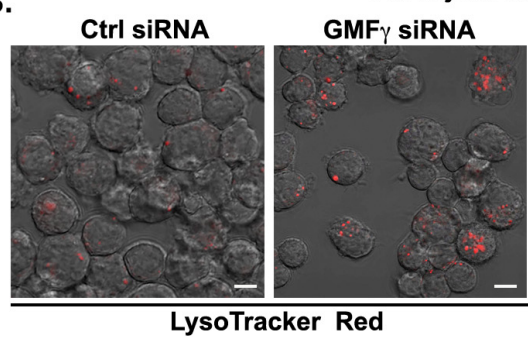

D.

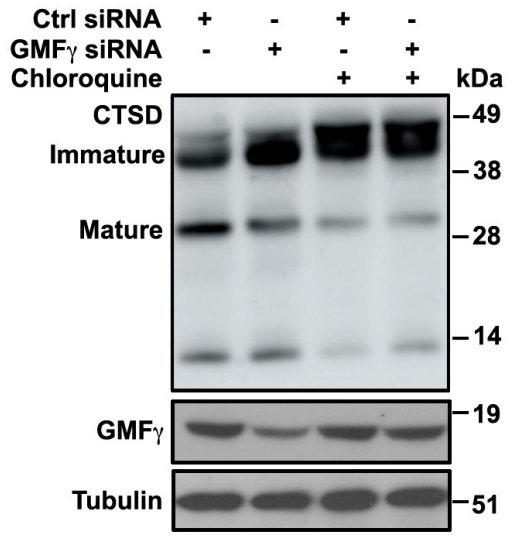


A.
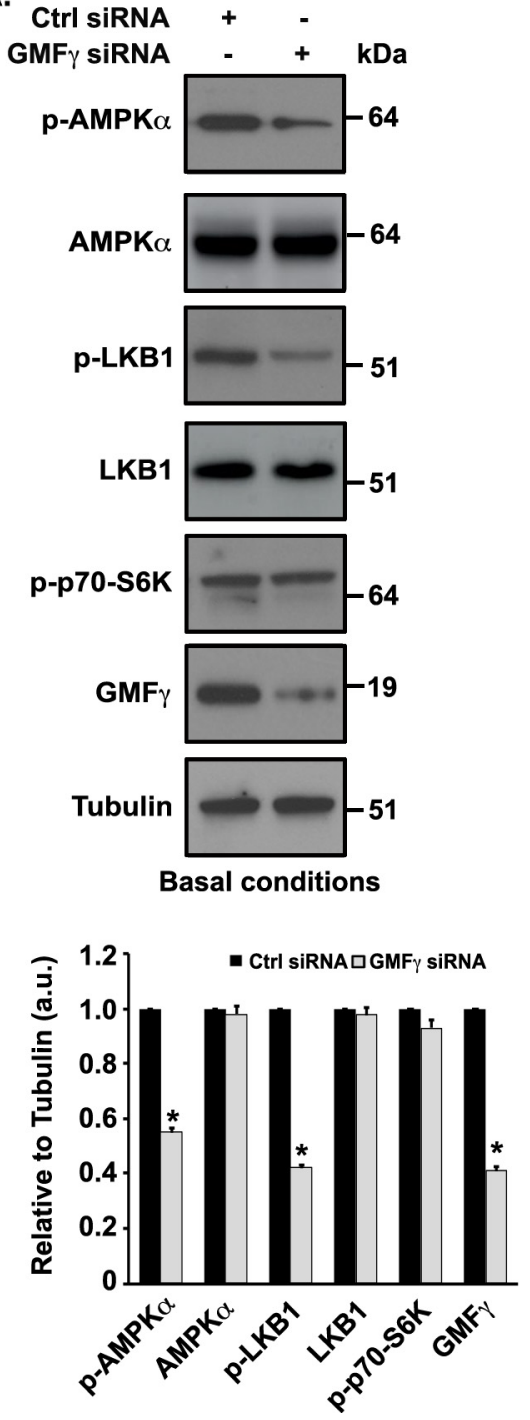

Aerbajinai et al.; Figure 3

B.

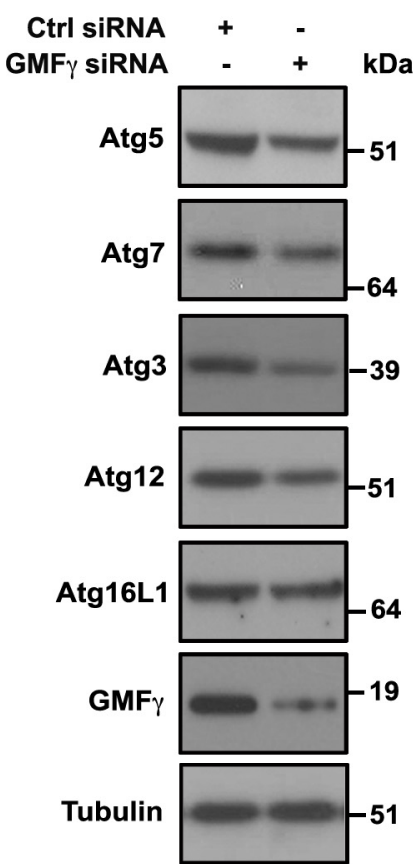

Basal conditions

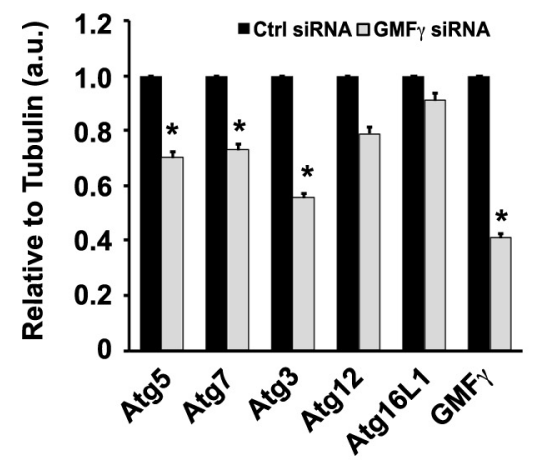



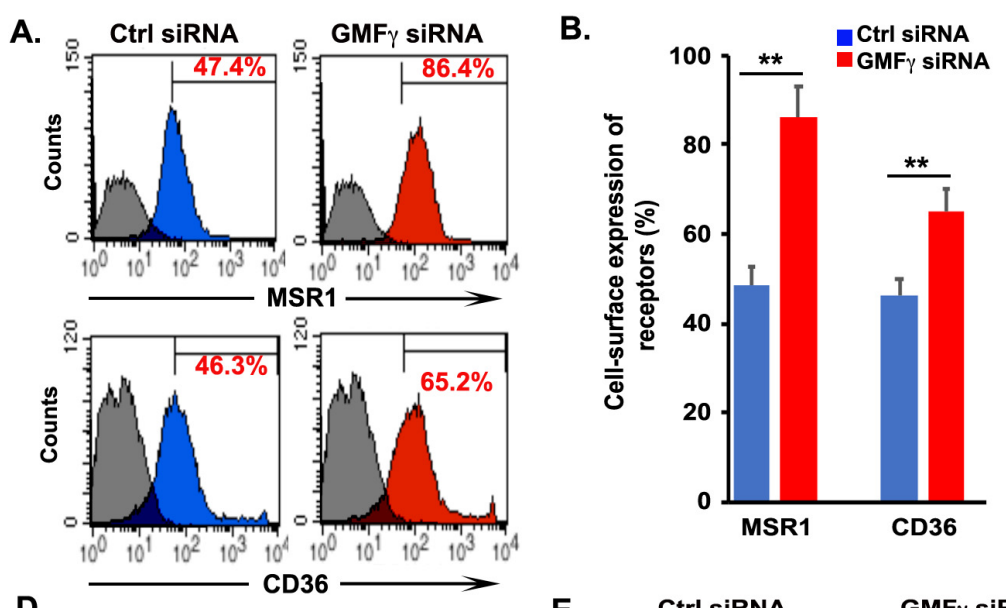

D.

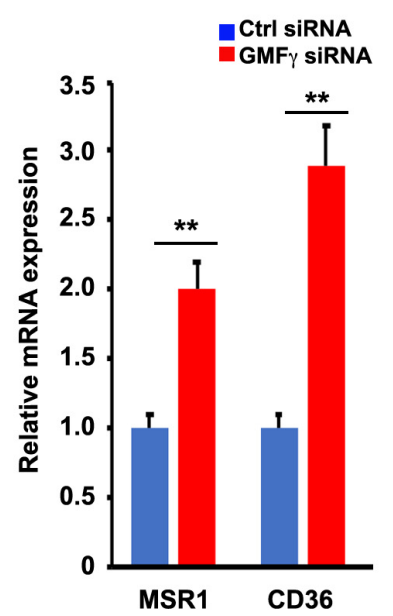

E.
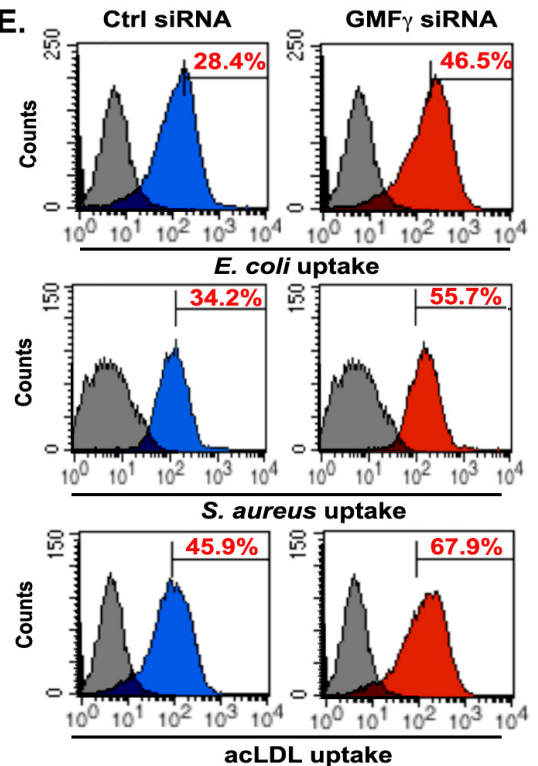

Aerbajinai et al.; Figure 4

C.

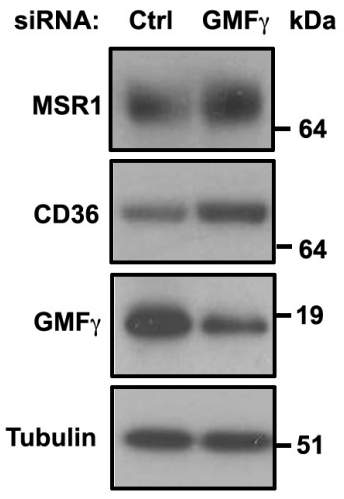

F.

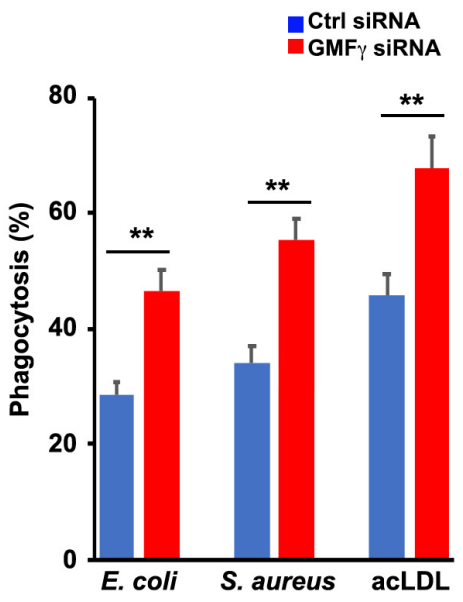


Aerbajinai et al.; Figure 5

A.

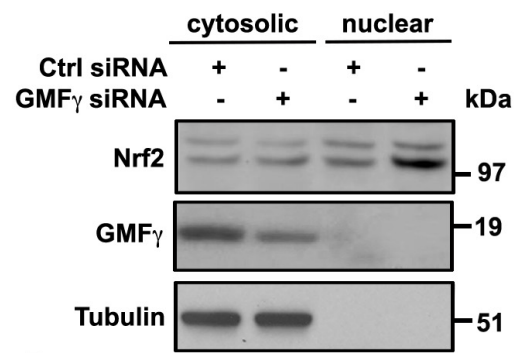

D.

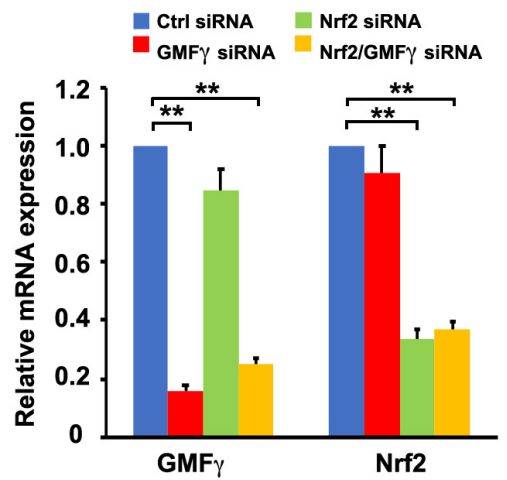

B.

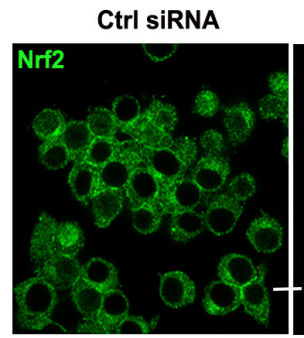

E.

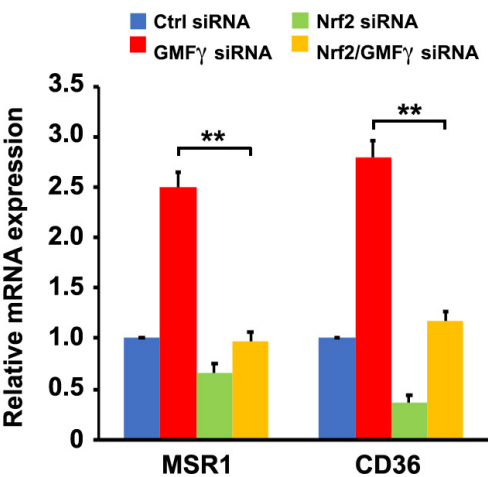

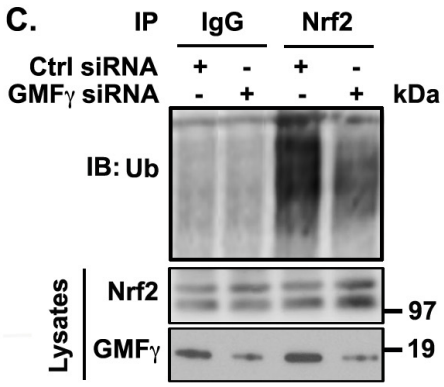

F.

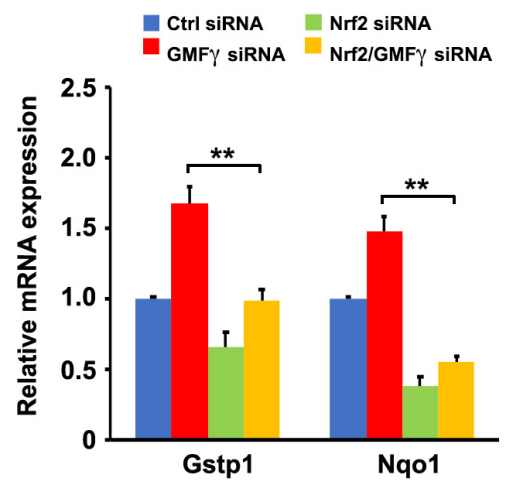


A.

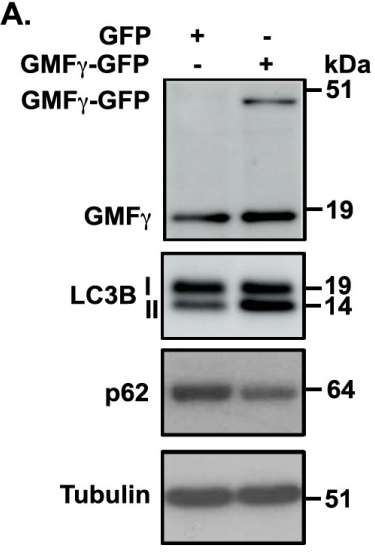

E.

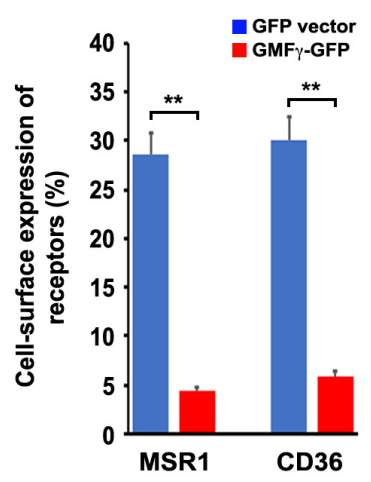

B.

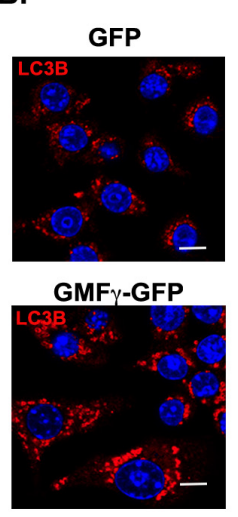

c.

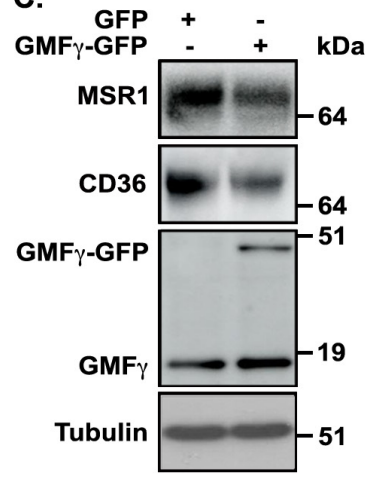

F.

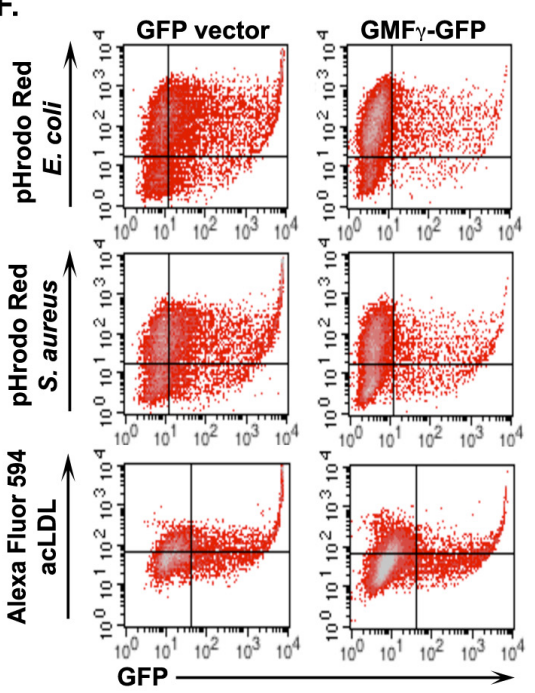

Aerbajinai et al.; Figure 6

D.
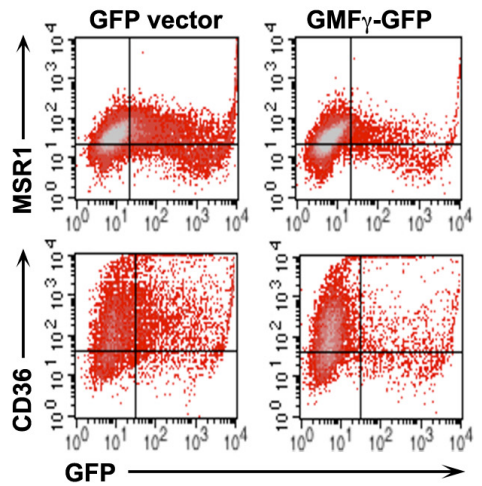

G.

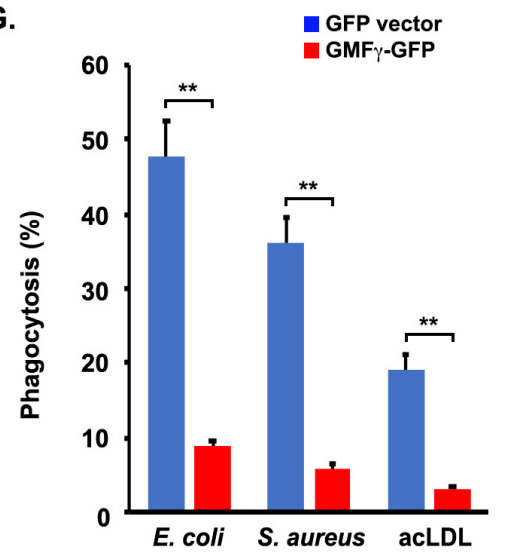


A.

Basal conditions

siRNA: Ctrl GMFy Ctrl GMFy kDa
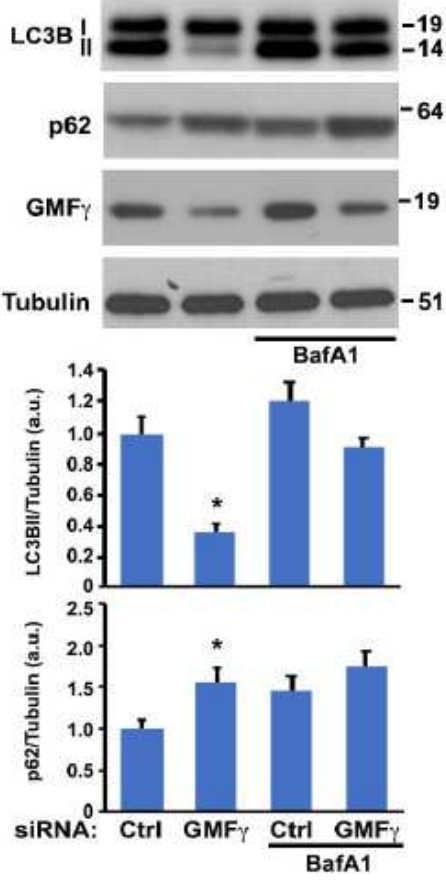

B.

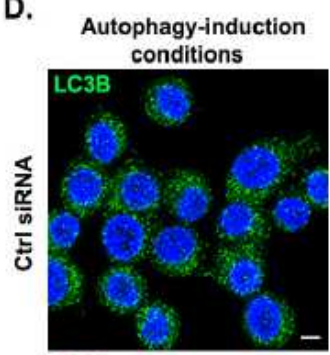

E.

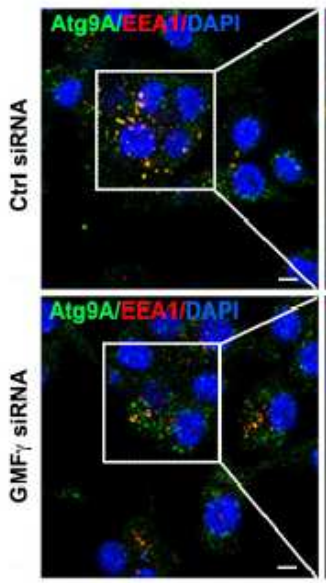

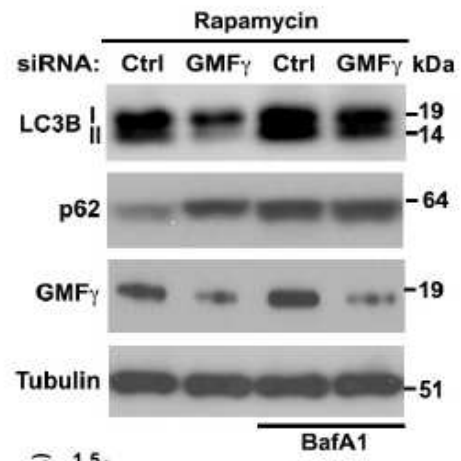
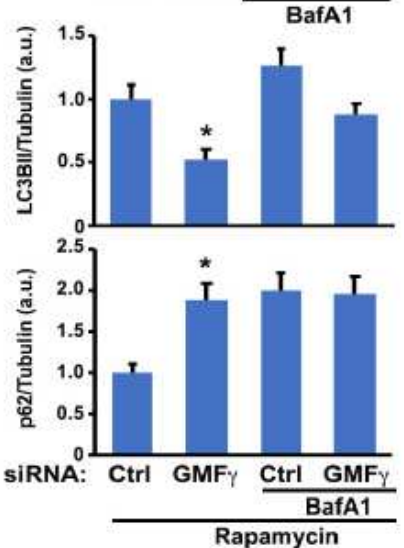

F.

F. Autophagy-induction

c.
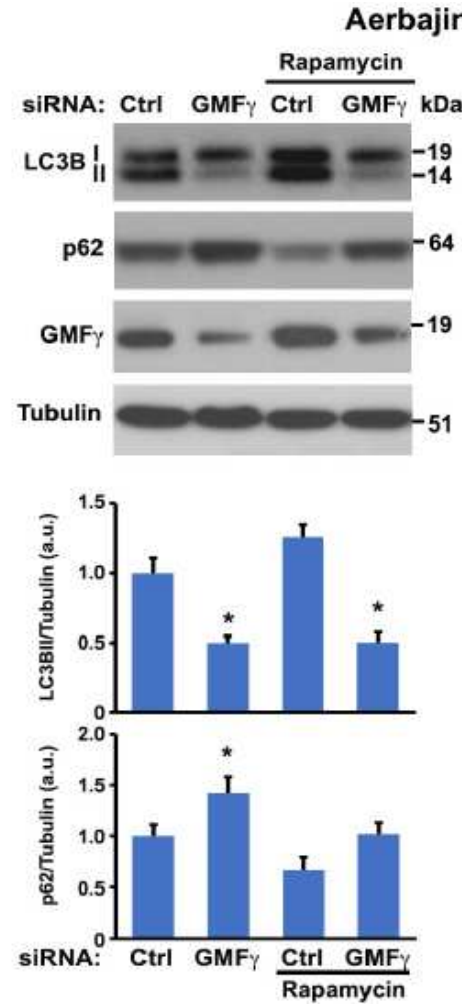

G.

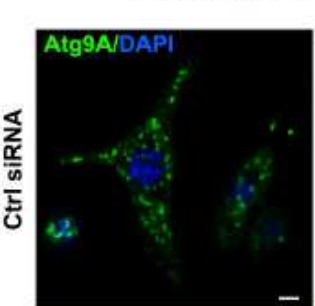

Autophagy-induction conditions
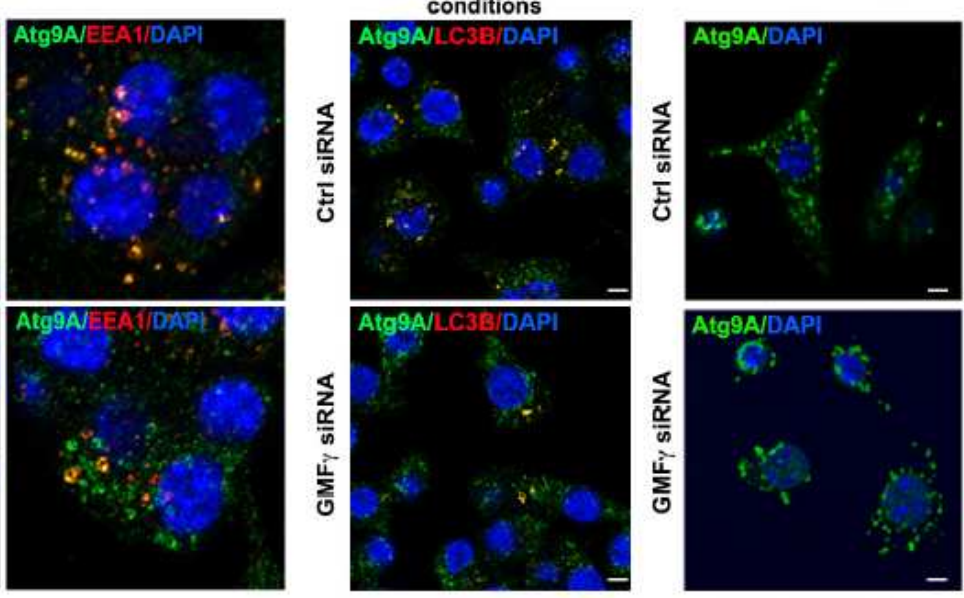
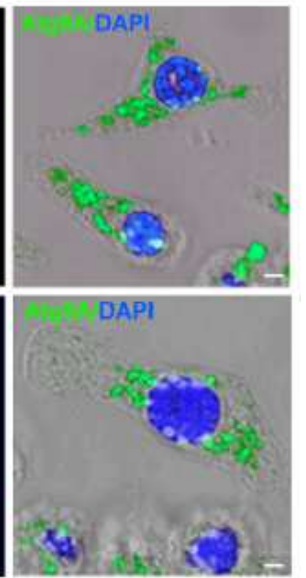

\section{Figure 1}

GMF y knockdown in murine macrophages impairs autophagosome formation. (A-C) RAW264.7 macrophages or BMDMs were transfected with a control negative siRNA (Ctrl siRNA) or GMF y siRNA for $48 \mathrm{~h}$. To induce autophagy, cells were stimulated with $4 \mu \mathrm{M}$ Rapamycin for $4 \mathrm{~h}$. Representative immunoblotting analysis of LC3B and p62 protein expression in lysates of RAW264.7 macropha treated with or without $300 \mathrm{nM}$ BafA1 for $4 \mathrm{~h}$ under basal (A) or autophagy-induction conditions (B) or BMDMs under basal and autophagy-induction conditions (C). Tubulin was used as a loading control. The graphs (lower) show relative quantification of LC3BII and p62 intensity from densitometric scans after normalizing to the control Tubulin in immunoblot (upper). Data represent the mean \pm S.D. of at least three 
independent experiments. Values for Ctrl siRNA-transfected cells were set to 1. *, $p<0.05$. (D) Representative immunofluorescence images of LC3B staining in Ctrl siRNA- or GMF y siRNA-transfected RAW264.7 macrophages under autophagy-induction conditions. Nuclear DNA was labeled with DAPI (blue). Images are representative of three independent experiments. Scale bars, $10 \mu \mathrm{m}$. (E and F) Representative immunofluorescence images of colocalization of Atg9A (anti-mouse Alexa Fluor 488) with EEA1 (anti-rabbit Alexa Fluor 568) under autophagy-induction conditions (E) or Atg9A (anti-mouse Alexa Fluor 488) with LC3B (anti-rabbit Alexa Fluor 568) under autophagy-induction conditions (F) in Ctrl siRNAor GMF y siRNA-transfected RAW264.7 macrophages. Nuclear DNA was labeled with DAPI (blue). Images are representative of three independent experiments. Boxed areas in middle column of images in $(E)$ are presented as enlarged images (2.5-fold) in the right-hand column. Scale bars, $10 \mu \mathrm{m}$. (G) Representative immunofluorescence images of Atg9A localization in Ctrl siRNA- or GMF y siRNAtransfected RAW264.7 macrophages under autophagy-induction conditions. Autophagy-induction conditions were produced by stimulating cells with $4 \mu \mathrm{M}$ Rapamycin for $4 \mathrm{~h}$. Nuclear DNA was labeled with DAPI (blue). Images are representative of three independent experiments. Scale bars, $10 \mu \mathrm{m}$.

A.

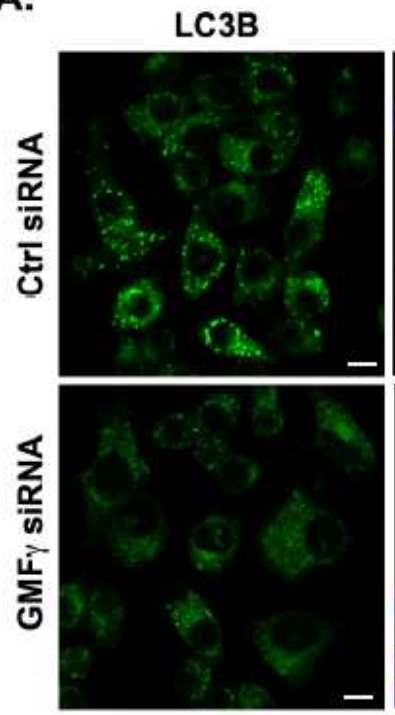

C.

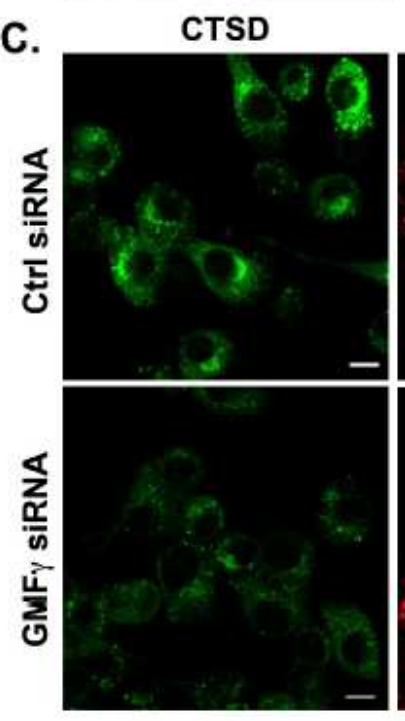

Lamp1
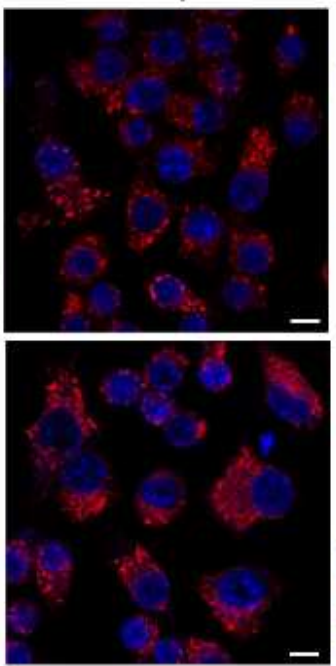

Lamp1

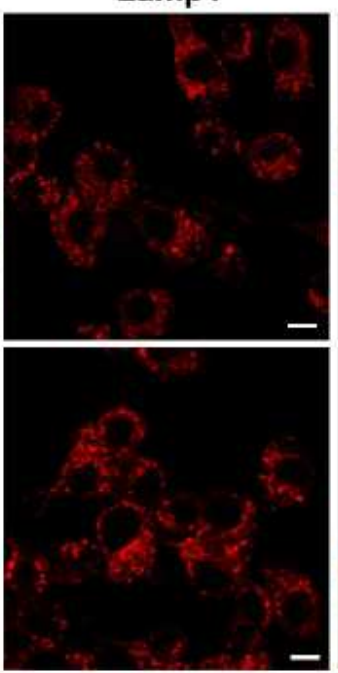

B.

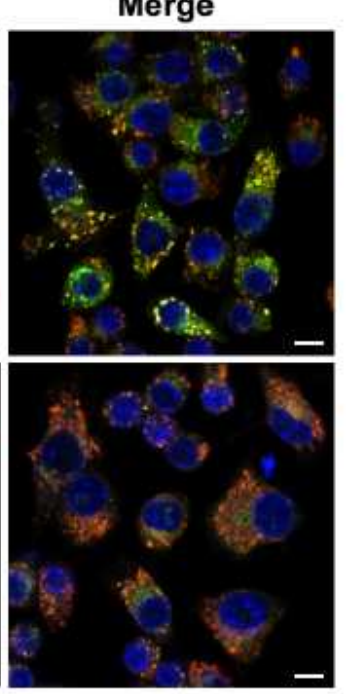

Merge

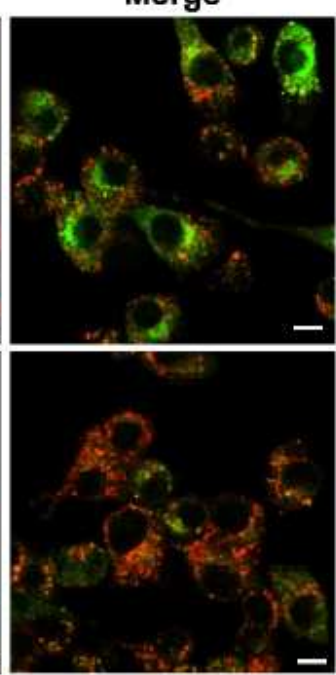

D.
Aerbajinai et al GMF $\gamma$ siRNA

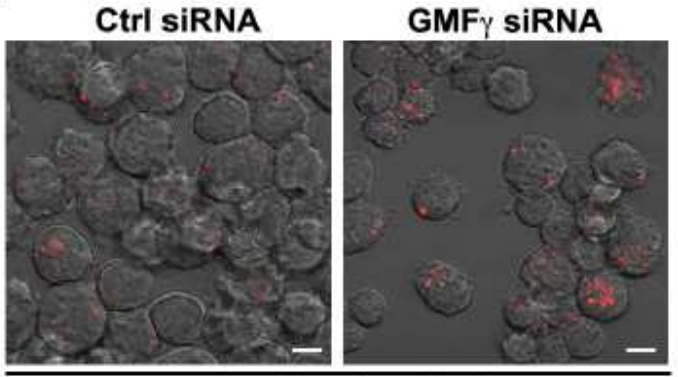

LysoTracker Red

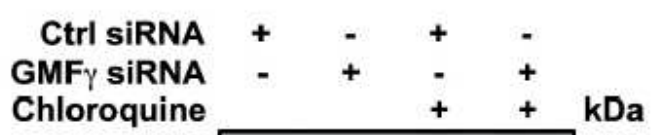

Chloroquine CTSD Immature $+-49$ $-38$

Mature

Mature $-28$

GMF $\gamma$

Tubulin

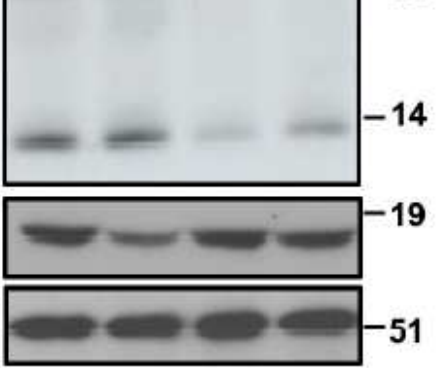

Figure 2 
GMF $y$ knockdown in murine macrophages inhibits lysosomal function. RAW264.7 macrophages were transfected with a control negative siRNA (Ctrl siRNA) or GMF y siRNA for $48 \mathrm{~h}$. (A) Representative immunofluorescence images of colocalization of LC3B (anti-mouse Alexa Fluor 488) with Lamp1 (antirabbit Alexa Fluor 568) in Ctrl siRNA- or GMF y siRNA-transfected macrophages under basal conditions. Nuclear DNA was labeled with DAPI (blue). Images are representative of three independent experiments. Scale bars, $10 \mu \mathrm{m}$. (B) Representative fluorescence images of Ctrl siRNA- or GMF Y siRNAtransfected cells treated with $75 \mathrm{nM}$ LysoTracker Red for $1 \mathrm{~h}$ under basal conditions. Images are representative of three independent experiments. Scale bars, $10 \mu \mathrm{m}$. (C) Representative immunofluorescence images of colocalization of CTSD (anti-mouse Alexa Fluor 488) with Lamp1 (antirabbit Alexa Fluor 568) in Ctrl siRNA- or GMF $y$ siRNA-transfected macrophages under basal conditions. Nuclear DNA was labeled with DAPI (blue). Images are representative of three independent experiments. Scale bars, $10 \mu \mathrm{m}$. (D) Representative immunoblotting analysis of CTSD maturation in lysates of Ctrl siRNA- or GMF y siRNAtransfected macrophages treated with or without $10 \mu \mathrm{M}$ chloroquine for $4 \mathrm{~h}$ under basal conditions. Left lane, molecular weight markers. Representative image from three independent experiments. Tubulin was used as a loading control. 
A.
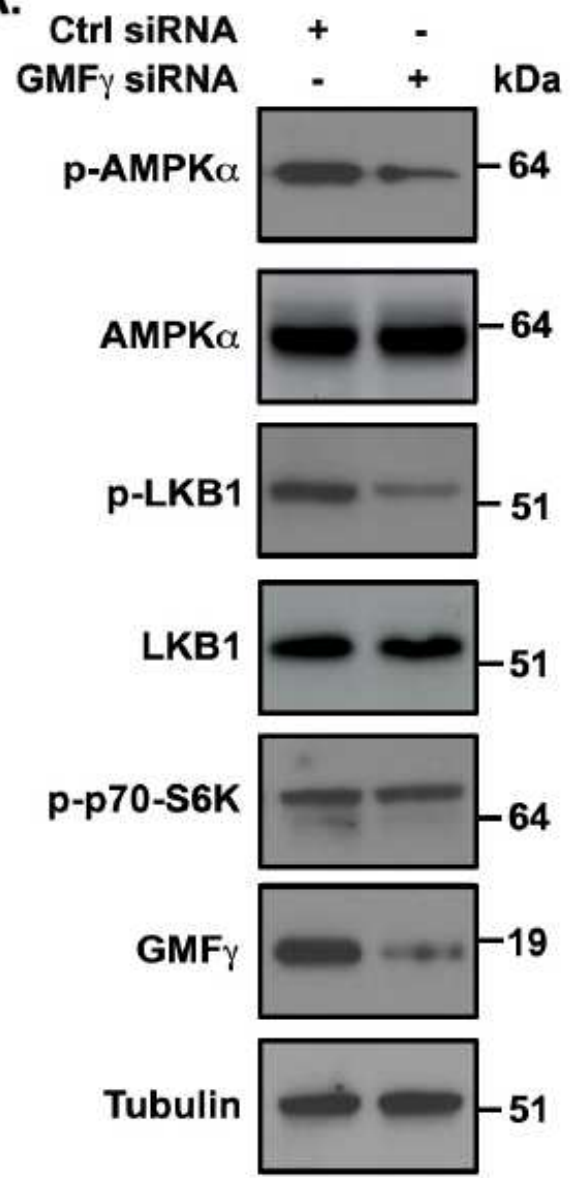

Basal conditions

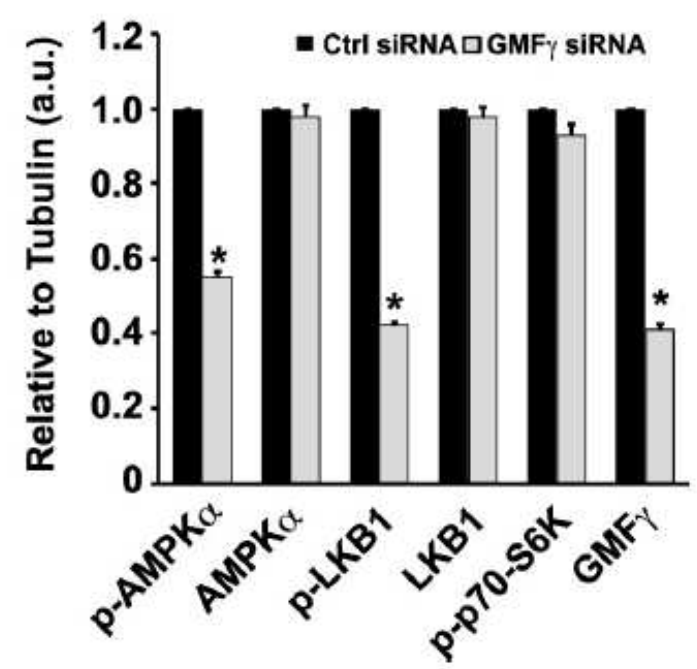

Aerbajinai et al.

B.
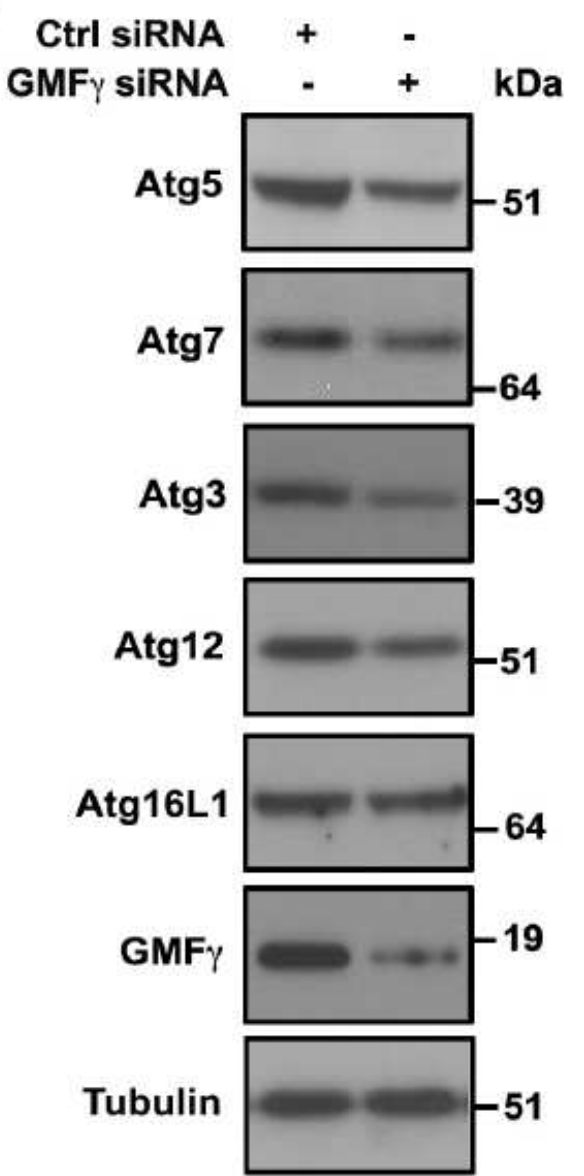

Basal conditions

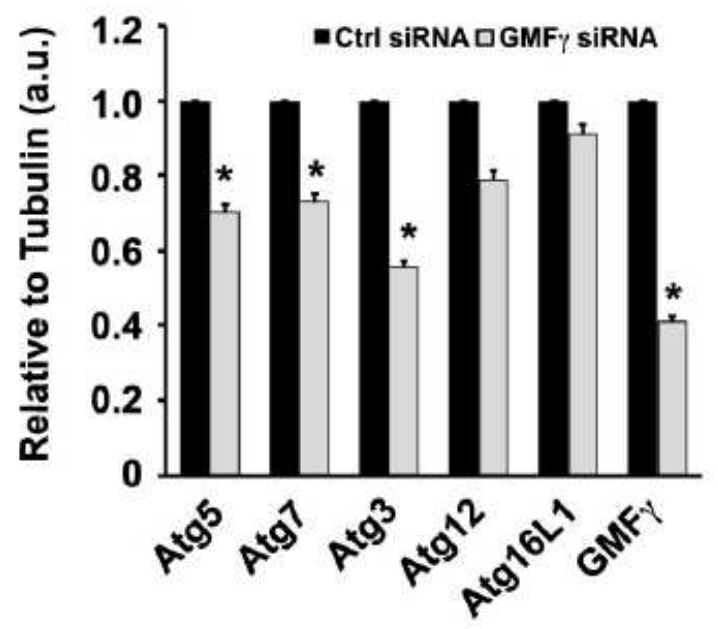

Figure 3

GMF $y$ knockdown in murine macrophages decreases phosphorylation of LKB1/AMPK and autophagy proteins. RAW264.7 macrophages were transfected with a control negative siRNA (Ctrl siRNA) or GMF Y siRNA for $48 \mathrm{~h}$. Representative immunoblotting analysis of LKB1/AMPK signaling pathway (A) and autophagy-related (B) protein expression in lysates of Ctrl siRNA- or GMF y siRNAtransfected macrophages under basal conditions. Tubulin was used as a loading control. Representative image from 
three independent experiments. Lower panels, histogram showing densitometry of the indicated protein levels relative to Tubulin (control) in Ctrl siRNA- or GMF y siRNA-transfected cells. Data represent the mean \pm S.D. of three independent experiments. Values for Ctrl siRNA-transfected cells were set to $1 . *, p<$ 0.05 compared with control cells.
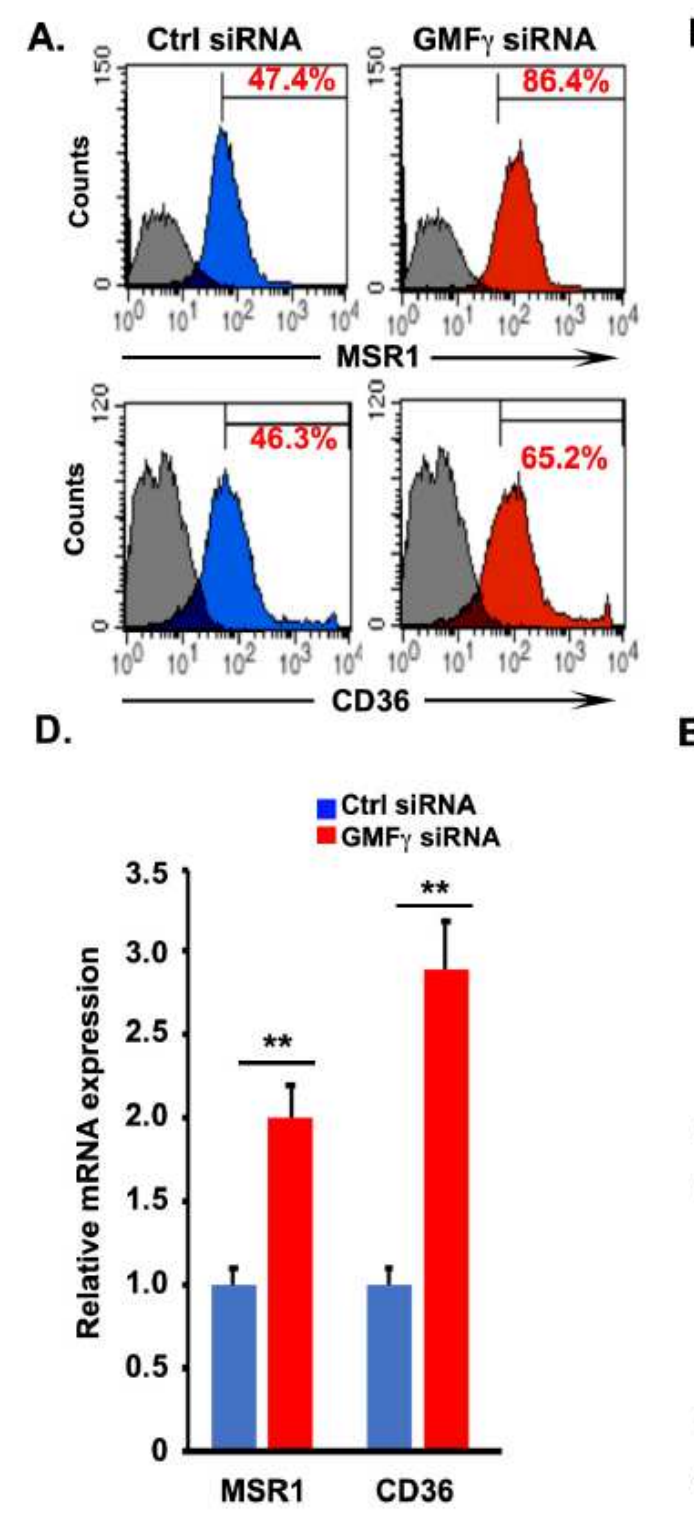

B.

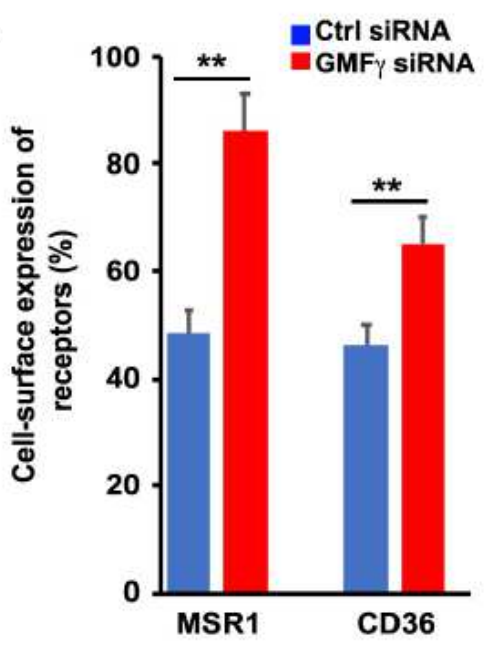

E.
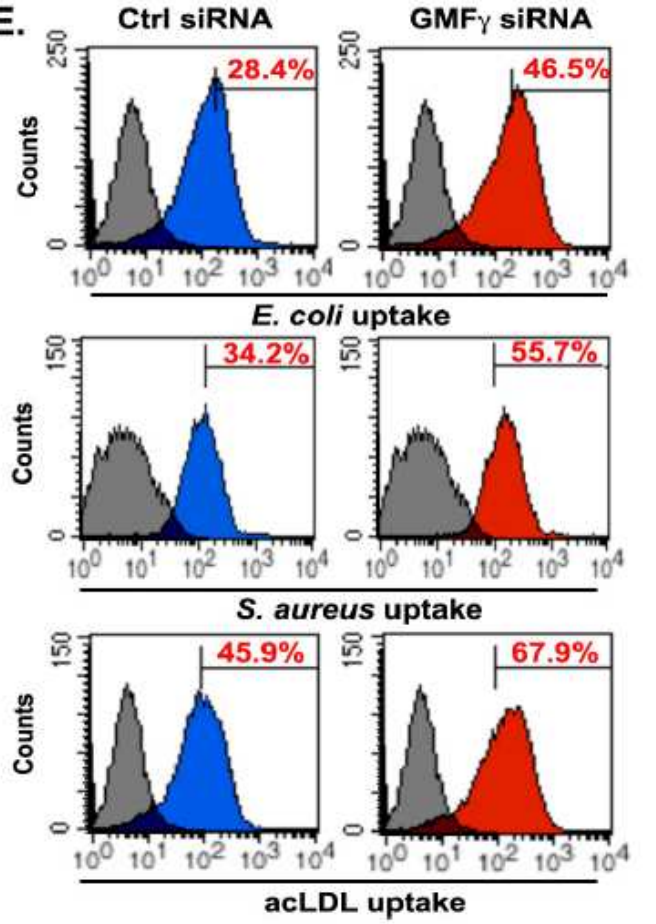

Aerbajinai et al.

c.

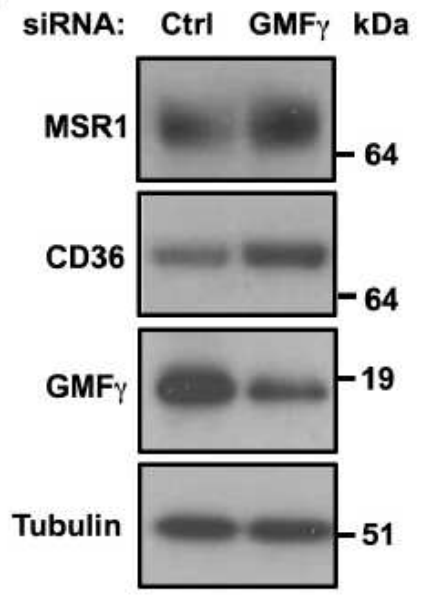

F.

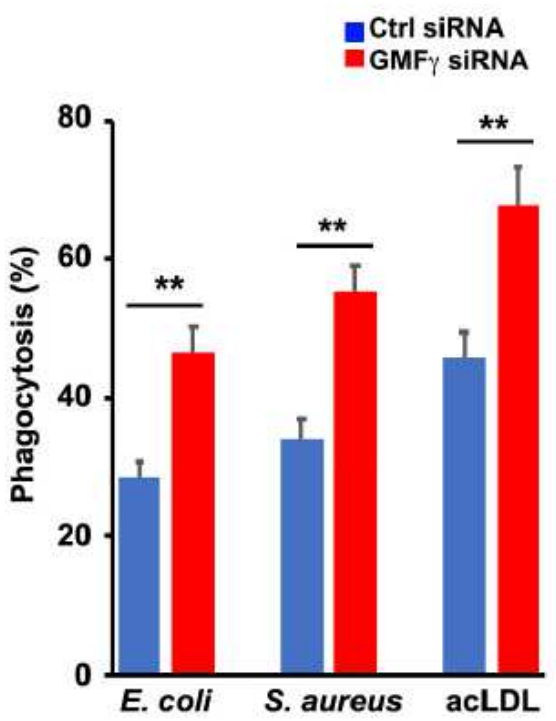

Figure 4

GMF y knockdown in murine macrophages increases scavenger-receptor expression and phagocytosis. RAW264.7 macrophages were transfected with a control negative siRNA (Ctrl siRNA) or GMF y siRNA for $48 \mathrm{~h}$. (A and B) Flow-cytometry analysis of cell-surface protein expression of MSR1 and CD36 in Ctrl siRNA- or GMF y siRNA-transfected macrophages. Representative histogram (A) and quantitative data (B) are shown. Data represent the mean \pm S.D. of three independent experiments. $* *, p<0.01$ compared with control cells. (C) Representative immunoblotting analysis of total cell protein expression of MSR1 and 
CD36 in lysates of Ctrl siRNA- or GMF y siRNA-transfected macrophages. Tubulin was used as a loading control. Representative image from three independent experiments. (D) QPCR analysis of MSR1 and CD36 mRNA expression in Ctrl siRNA- or GMF y siRNA-transfected macrophages. Data are expressed as fold increase, normalized to $18 \mathrm{~S} \mathrm{mRNA}$, and represent the mean \pm S.D. of three independent experiments. Values for Ctrl siRNA-transfected cells were set to 1. ${ }^{*}, p<0.01$ compared with control cells. (E and $F$ ) Phagocytosis of E. coli, S. aureus, and acetylated LDL (acLDL) in Ctrl siRNA- or GMF y siRNA-transfected macrophages. Flow cytometry analyzes phagocytic uptake of Alexa Fluor 488-conjugated E. coli (K-12 strain), pHrodo ${ }^{\mathrm{TM}}$ Green S. aureus BioparticlesR, or Alexa Fluor 488-conjugated acetylated LDL after 45 min. Representative histogram (E) and quantitative data (F) are shown. Data represent the mean phagocytosis percentage \pm S.D. of three independent experiments. ${ }^{* *}, p<0.01$ compared with control cells.

A.

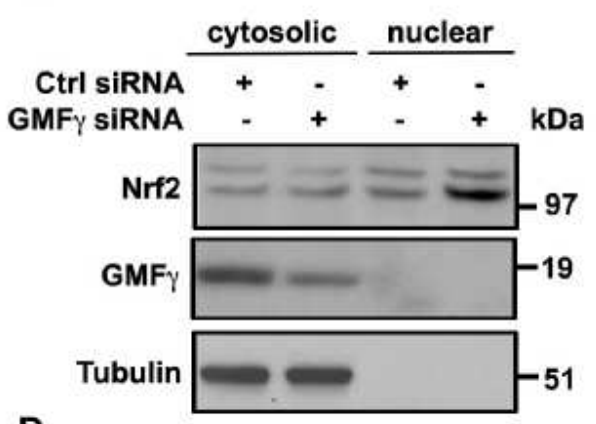

D.

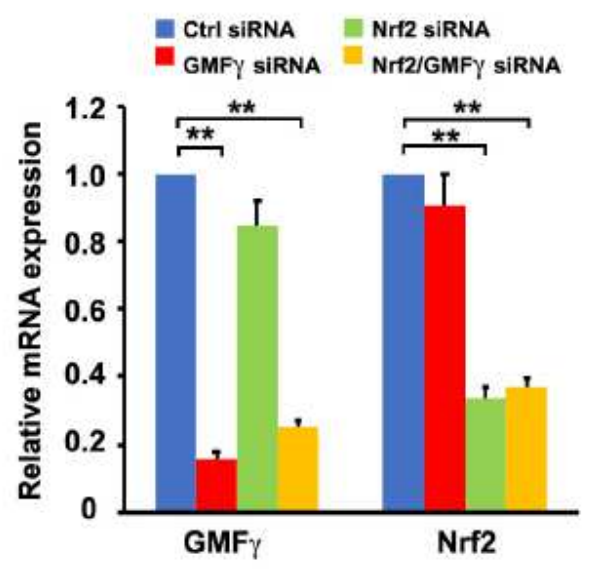

B.

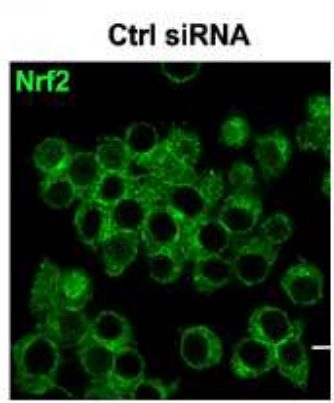

E.

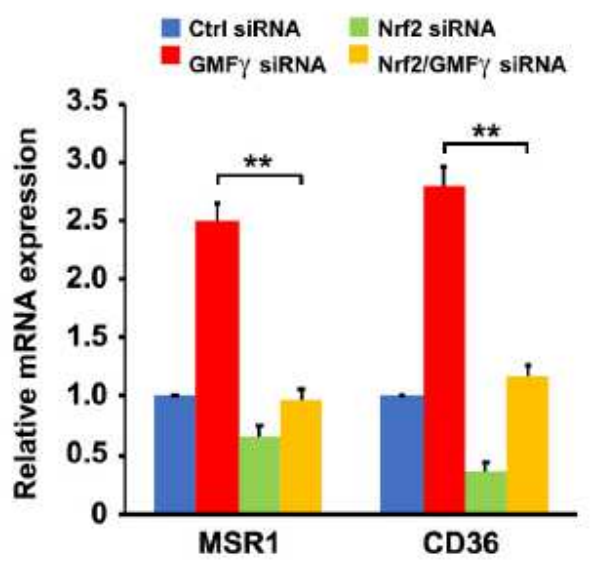

Aerbajinai et al.

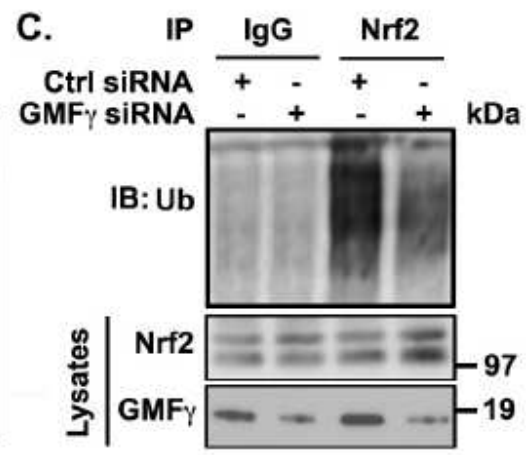

F.

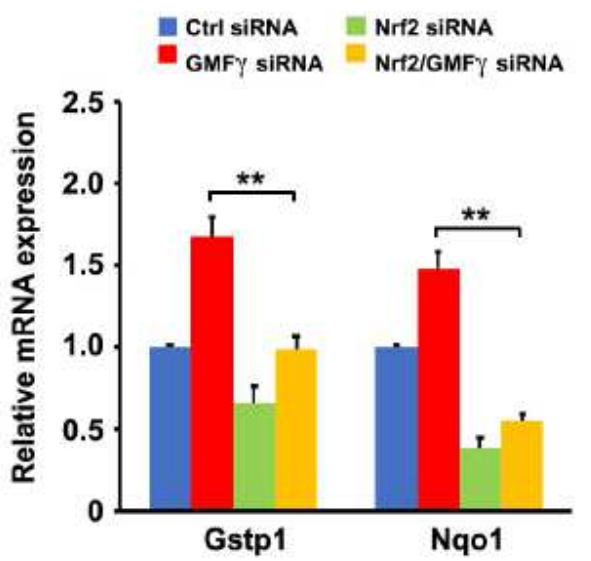

Figure 5

GMF y knockdown in murine macrophages promotes Nrf2 nuclear translocation and increases scavenger-receptor expression by a Nrf2-dependent mechanism. (A-C) RAW264.7 macrophages were transfected with a control negative siRNA (Ctrl siRNA) or GMF y siRNA for $48 \mathrm{~h}$. (A) Representative immunoblotting analysis of Nrf2 protein expression in cytosolic and nuclear extracts of Ctrl siRNA- or GMF y siRNA-transfected macrophages. Tubulin were used as a loading control. Representative image from three independent experiments. (B) Representative immunofluorescence images of Nrf2 nuclear translocation in Ctrl siRNA- or GMF y siRNA-transfected macrophages. Images are representative of three independent experiments. Scale bar, $10 \mu \mathrm{m}$. (C) Representative immunoprecipitation analysis of Nrf2 
ubiquitination in Ctrl siRNA- or GMF y siRNA-transfected macrophages. Endogenous Nrf2 was immunoprecipitated (IP) from transfected cell lysates with anti-Nrf2 (or nonspecific IgG, as a negative control) and examined for ubiquitination by immunoblotting (IB) with anti-ubiquitin or anti-Nrf2 antibodies. Representative image from two independent experiments. (D-F) RAW264.7 macrophages were transfected with a Ctrl siRNA, GMF y siRNA, or Nrf2 siRNA for $48 \mathrm{~h}$. or, for GMF $\mathrm{y}+\mathrm{Nrf2}$ doubleknockdown, with Nrf2 siRNA for $24 \mathrm{~h}$ followed by transfection with GMF y siRNA for another $48 \mathrm{~h}$. Q-PCR analysis of GMF $y$ and Nrf2 (D), MSR1 and CD36 (E), or Gstp1 and Nqo1 (F) mRNA expression in Ctrl siRNA-, GMF y siRNA-, Nrf2 siRNA-, or Nrf2 siRNA + GMF y siRNA-transfected macrophages. Data are expressed as fold increase, normalized to $18 \mathrm{~S} \mathrm{mRNA}$, and represent the mean \pm S.D. of three independent experiments. Values for Ctrl siRNA-transfected cells were set to $1 .{ }^{\star *} p<0.01$.

A.

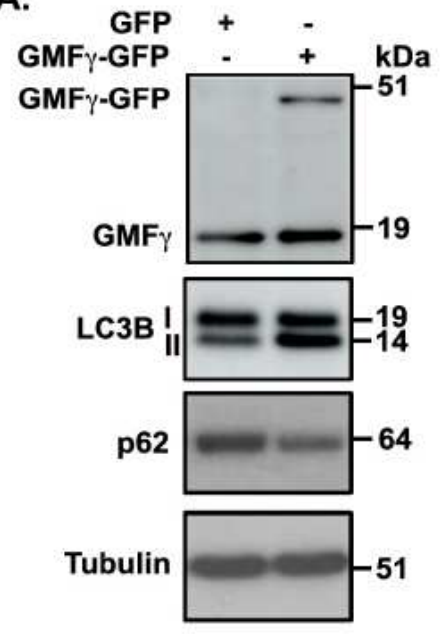

E.

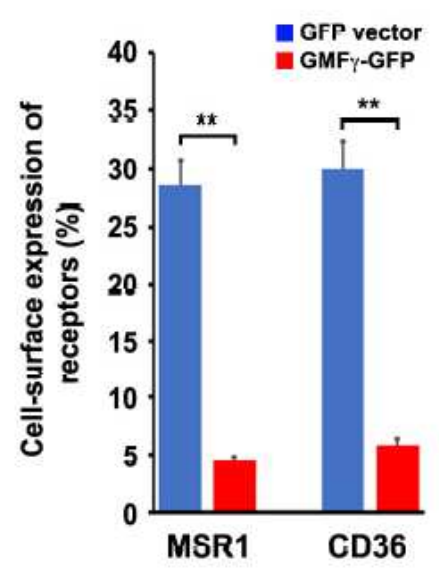

B.

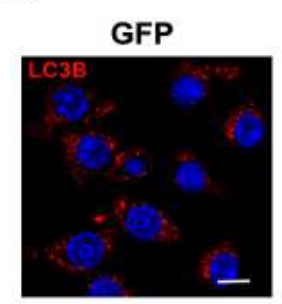

GMF $\gamma$-GFP

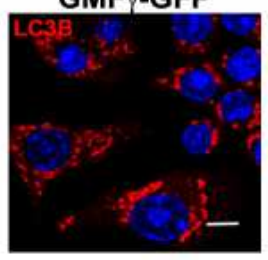

C.

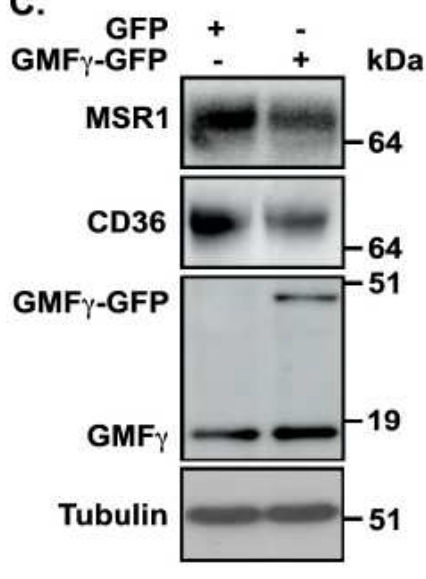

F.

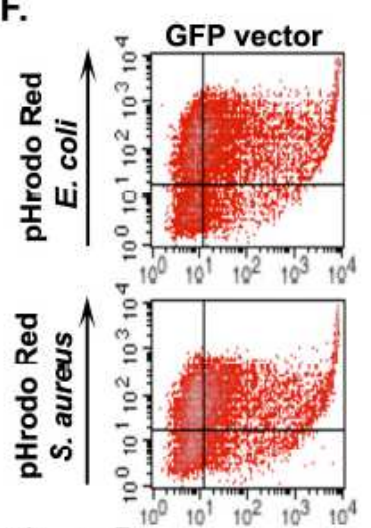

壳
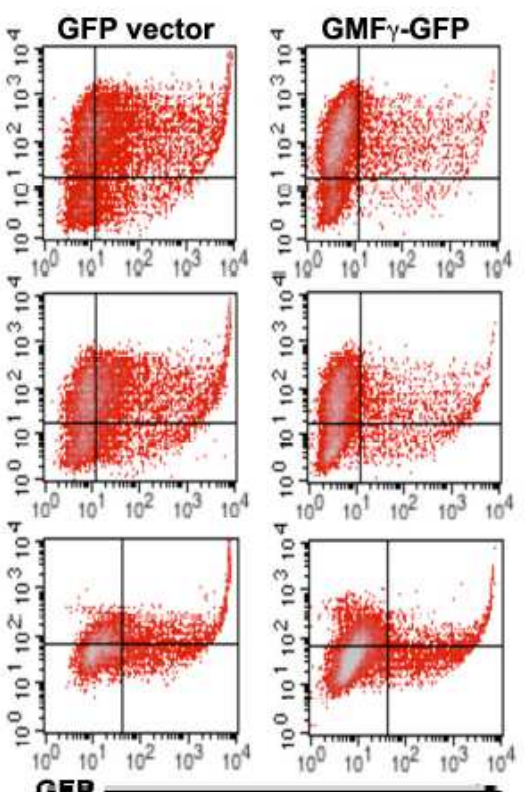

Aerbajinai et al.

D.
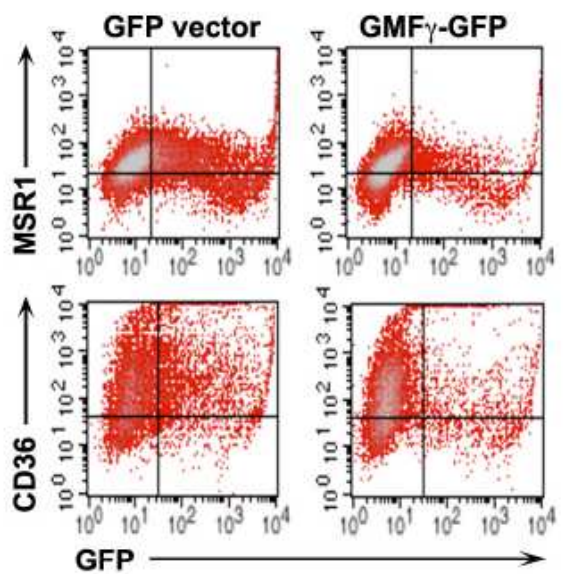

G.

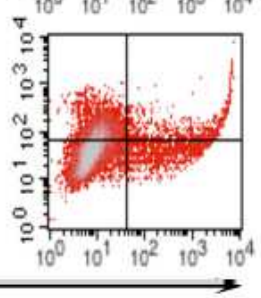

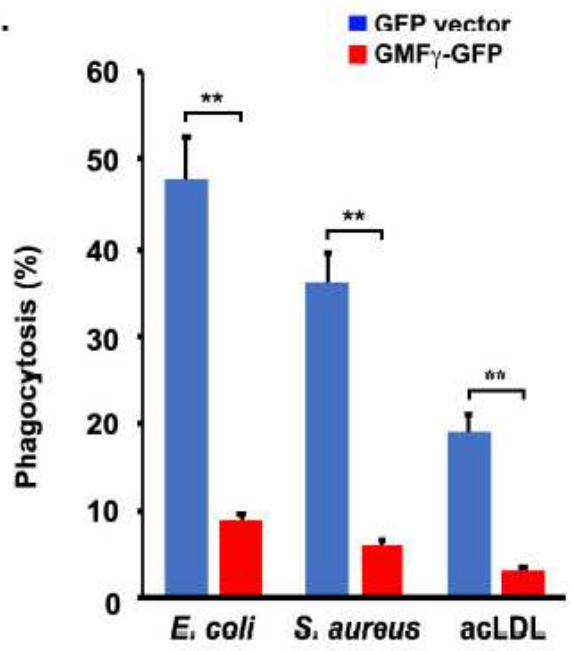

Figure 6

GMFy overexpression in murine macrophages increases autophagosome formation and decreases phagocytosis. RAW264.7 macrophages were transfected with GMFY-GFP or GFP control plasmid for $24 \mathrm{~h}$. 
(A) Representative immunoblotting analysis of GMFy-GFP, GMFy, LC3B, and p62 protein expression in lysates of GFP control plasmid- or GMFY-overexpressing macrophages. Representative image from three independent experiments. Tubulin was used as a loading control. (B) Representative immunofluorescence images of LC3B staining in GFP control plasmid- or GMFy overexpressing macrophages. Nuclear DNA was labeled with DAPI (blue). Images are representative of three independent experiments. Scale bar, 10 $\mu \mathrm{m}$. (C) Representative immunoblotting analysis of MSR1 and CD36 protein expression in lysates of GFP control plasmid- or GMFy-overexpressing macrophages. Representative image from three independent experiments. Tubulin was used as a loading control. (D and E) Flow-cytometry analysis of cell-surface expression levels of MSR1 and CD36 in GFP control plasmidor GMFY-overexpressing macrophages.

Representative histogram (D) and quantitative data (E) are shown. Data represent the mean . S.D. of three independent experiments. ${ }^{*}, \mathrm{p}<0.01$ compared with GFP control plasmid cells. ( $F$ and $G$ ) Phagocytosis of E. coli, S. aureus, and acetylated LDL (acLDL) in GFP control plasmid- or GMFY-GFP-overexpressing macrophages. Flow cytometry analyzes phagocytic uptake of pHrodo $^{\text {TM }}$ Red E. coli Bioparticles ${ }^{T M}$, pHrodo ${ }^{\text {TM }}$ Red S. aureus Bioparticles ${ }^{\mathrm{TM}}$, or Alexa Fluor 594- conjugated acetylated LDL after $45 \mathrm{~min}$. Representative histogram $(F)$ and quantitative data $(G)$ are shown. Data represent the mean phagocytosis percentage \pm S.D. of three independent experiments. ${ }^{*}, \mathrm{p}<0.01$ compared with GFP control plasmid cells. 